\title{
Stresses and Liftings of Cell-Complexes
}

\author{
K. Rybnikov \\ Department of Mathematics and Statistics, Queen's University, \\ Kingston, Ontario, Canada K7L 3N6 \\ rybnikov@mast.QueensU.CA
}

\begin{abstract}
This paper introduces a general notion of stress on cell-complexes and reports on connections between stresses and liftings (generalization of $C_{1}^{0}$-splines) of $d$-dimensional cell-complexes in $\mathbb{R}^{d}$. New sufficient conditions for the existence of a sharp lifting for a "flat" piecewise-linear realization of a manifold are given. Our approach also gives some new results on the equivalence between spherical complexes and convex and star polytopes. As an application, two algorithms are given that determine whether a piecewise-linear realization of a $d$-manifold in $\mathbb{R}^{d}$ admits a lifting to $\mathbb{R}^{d+1}$ which satisfies given constraints. We also demonstrate connections between stresses and Voronoi-Dirichlet diagrams and show that any weighted Voronoi-Dirichlet diagram without non-compact cells can be represented as a weighted Delaunay decomposition and vice versa.
\end{abstract}

\section{Introduction}

In this paper we develop a variety of geometrical and algorithmic methods that are useful for studying piecewise-linear surfaces, weighted Voronoi and Delaunay diagrams, self-stresses in frameworks and geometrical cell-complexes and parallel drawings of polyhedral pictures. The space of $d$-stresses on a cell-complex introduced in this paper plays an important role in investigations of many affine and projective properties of a broad class of geometrical cell-complexes. Our notion of stress generalizes the notion of affine stress introduced by Lee [26].

In Sections 3-7 we prove that for a piecewise-linear realization in $\mathbb{R}^{d}$ of a homology manifold $\mathcal{M}^{d}$ with $H_{1}\left(\mathcal{M}^{d}, \mathbb{Z}_{2}\right)=0$, and for an arbitrary decomposition of $\mathbb{R}^{d}$ by convex polyhedra, the linear space of $d$-stresses is isomorphic to:

1. the linear space of liftings (with one fixed $d$-cell);

2. the linear space of reciprocals (with one fixed vertex).

These results can be considered as generalizations of similar results by Crapo and 
Whiteley for $d=2,3$ [12]-[14], [48]. For the first time this equivalence is proved under the general condition $H_{1}\left(\mathcal{M}^{d}, \mathbb{Z}_{2}\right)=0$ for $d>3$. Thus, there are non-spherical closed compact manifolds for which the equivalence between stresses and liftings holds. If $\Delta$ is a decomposition of $\mathbb{R}^{d}$ by convex polyhedra, then the cone of $d$-tensions is equivalent to: (1) the cone of additively weighted Voronoi-Dirichlet diagrams, representing $\Delta$; (2) the cone of convex liftings; (3) the cone of convex reciprocals. The equivalence between the last three objects was proved earlier by Aurenhammer [4] and McMullen [32]. In Section 8 we consider a general notion of duality for decompositions of $\mathbb{R}^{d}$ by convex polyhedra, and show that the classes of weighted Voronoi and Delaunay diagrams (decompositions) coincide.

New sufficient conditions for the existence of a sharp lifting of a piecewise-linear realization of a cell-decomposition of a manifold in $\mathbb{R}^{d}$ are given in Sections 8 and 9. For instance, we show that any closed $(d-3)$-primitive manifold $\mathcal{M}^{d}$ (where the star of each $(d-3)$-cell has only four $d$-cells) with $H_{1}\left(\mathcal{M}^{d}, \mathbb{Z}_{2}\right)=0$ has a sharp lifting, but the existence of a sharp lifting for a $(d-2)$-primitive manifold $\mathcal{M}^{d}$ (where the star of each internal $(d-3)$-cell has only three $d$-cells) with $H_{1}\left(\mathcal{M}^{d}, \mathbb{Z}_{2}\right)=0$ requires the existence of a non-trivial $d$-stress on the star of each $(d-3)$-cell. In the same sections the problems of convexity and uniqueness for liftings are analysed. These results are improvements upon the well-known theorem of Davis [15] on the existence and uniqueness of a sharp convex lifting for a simple cell-decomposition of $\mathbb{R}^{d}, d>2$, and upon similar results of Crapo and Whiteley on liftings of simple piecewise-linear spheres [14], [50]. As an application of the developed geometrical approach, two algorithms are given that determine whether a piecewise-linear realization of a $d$-manifold in $\mathbb{R}^{d}$ admits a lifting to $\mathbb{R}^{d+1}$, which satisfies given constraints, and find the dimension of the space of liftings. For decompositions of $\mathbb{R}^{d}$ by convex polyhedra, often referred to as tilings, these algorithms also recognize whether a decomposition is a weighted Voronoi-Dirichlet diagram and determine whether there is a convex surface which projects onto this decomposition. The first algorithm (Section 11) applies only to $(d-2)$-primitive manifolds, and has linear running time in the number of $(d-1)$-faces, which is optimal. This algorithm is similar to an algorithm of Aurenhammer [3], but is more general and can be applied to a broader class of complexes. The second algorithm (Section 12) applies to general celldecompositions of homology manifolds (including arbitrary cell-decompositions of $\mathbb{R}^{d}$ ), but has worse time complexity, although it is polynomial. All theorems and algorithms are also interpreted for spherical cell-complexes (Section 13). These interpretations give new criteria and algorithms for recognizing whether a spherical complex is the radial projection of a convex polytope.

\section{Polyhedral Cell-Complexes}

All complexes which we consider are simplicial complexes from the topological point of view. However, all theorems and algorithms in this paper are stated for fixed decompositions of simplicial complexes into polyhedral cells (also called blocks or simplicial stars in combinatorial topology, see [27] and [34]) which are not necessarily simplexes. We assume that all complexes have at most a countable number of cells and are locally finite. A homology $k$-sphere ( $k$-disk) is a polyhedron with the homology groups of a 
standard $k$-sphere ( $k$-disk). A compact $k$-cell is understood to be a polyhedron (simplicial complex) which is a cone with a homology $k$-sphere as base. Cells of co-dimension 1 are referred to as facets. To include into consideration general cell-decompositions of Euclidean spaces into convex polyhedra we allow non-compact cells in a realization of a cell-complex in $\mathbb{R}^{d}$; i.e. a polyhedral $k$-dimensional subset of the realization may be considered as a whole cell from the geometrical point of view, if it lies in a $k$-dimensional affine subspace, and is homeomorphic to a linear half-space of $\mathbb{R}^{k}$. We also assume that each non-compact cell has finitely many faces.

We denote the star of a $k$-dimensional cell $C^{k}$ by $S t\left(C^{k}\right)$, and the $k$-dimensional skeleton of a complex $\mathcal{K}^{d}$ by $S k^{k}\left(\mathcal{K}^{d}\right)$. For us the relative boundary $\partial \mathcal{K}^{d}$ of a complex $\mathcal{K}^{d}$ is a sub-complex of $\mathcal{K}^{d}$ which consists of the closures of all $(d-1)$-cells which are not shared by at least two $d$-cells. We refer to cells that belong to the relative boundary as boundary cells, and to the other cells as internal. We denote the number of $k$-cells of $\mathcal{K}^{d}$ by $f_{k}\left(\mathcal{K}^{d}\right)$ and the number of internal $k$-cells by $f_{k}^{\circ}\left(\mathcal{K}^{d}\right)$. A (combinatorial) path in a complex $\mathcal{K}^{d}$ is a finite ordered sequence $\mathfrak{p}=\left[C_{1}, \ldots, C_{k}\right]$ of $d$-cells, where every two consecutive $d$-cells share a common $(d-1)$-cell. A circuit is a path where the first and last cells coincide.

We consider a somewhat more general construction than an embedding or an immersion of a cell-complex into Euclidean space, such as a piecewise-linear $(P L-)$ realization of a cell-complex in Euclidean space. In all geometric discussions cell-complexes will be considered as fixed PL-realizations, rather than abstract combinatorial objects.

Such a general construction can be helpful, for example, for studying frameworks with bar intersections, polyhedral scenes, Schlegel diagrams, and splines over triangulations (in the planar case this point of view was adopted in [12], [45], [47], and [49]; in the three-dimensional case such PL-realizations were considered by Crapo and Whiteley in [12] and [48]). For example, a Schlegel $d$-diagram (see Ziegler's book [52] for the theory of Schlegal diagrams) is a PL-realization of a $(d+1)$-polytope $P^{d}$ in $\mathbb{R}^{d}$ obtained by radial projection of $P^{d}$ onto one of its facets (a Schlegel diagram of a 4-cube is drawn in Fig. 1).

One can identify an abstract combinatorial cell-complex $\mathcal{K}^{d}$ with its embedding into $\mathbb{R}^{2 d+1}$ (since it can be triangulated). A PL-realization of a combinatorial simplicial

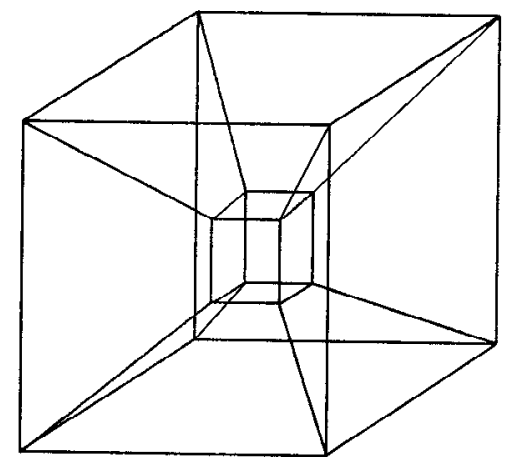

Fig. 1. A Schlegel diagram of a 4-cube (a PL-realization of a 4-cube in $\mathbb{R}^{3}$ ). 
complex $\mathcal{K}^{d} \subset \mathbb{R}^{2 d+1}$ with a fixed decomposition into polyhedral cells is a continuous PL-mapping $r$ of $\mathcal{K}^{d}$ in $\mathbb{R}^{N}(N \geq d)$ such that the closure of each $k$-cell, $k=0, \ldots, d$ is embedded by $r$ into $\mathbb{R}^{N}$ as a "flat" (lying in a $k$-subspace) $k$-polyhedron. For a PLrealization $\left(\mathcal{K}^{d}, r\right)$, we reserve an upper case roman font $K^{d}=\left(\mathcal{K}^{d}, r\right)$, and for an abstract combinatorial structure an upper case script $\mathcal{K}^{d}$. The body of $r\left(\mathcal{K}^{d}\right)$ is denoted by $\left|K^{d}\right|$.

If we refer to the metric, projective or affine properties of a cell-complex, these should be understood as the properties of its fixed PL-realization. However, when we consider the combinatorial or homological properties of a cell-complex, we are referring to its abstract combinatorial structure. A PL-realization of the star of a $k$-cell in $\mathbb{R}^{d}$ is called generic (for us) if all its $(d-1)$-cells lie on different $(d-1)$-planes.

We consider only strongly connected, pure dimensional complexes. A homology $d$-manifold (with boundary) is a cell-complex such that the link of each $k$-cell, $k=$ $0, \ldots, d-1$, is either a homology $(d-k-1)$-sphere or a homology $(d-k-1)$ disk. A manifold is closed if each facet is adjacent to exactly two $d$-cells. All statements in the paper are formulated for both closed manifolds and for manifolds with a boundary, unless stated otherwise. Since we consider manifolds only from the combinatorial point of view, a manifold is always understood to be a homology manifold.

The star of an internal $k$-cell in a $d$-manifold is called $(d-k)$-primitive if it has $d-k+1 d$-cells. A cell-decomposition of a $d$-manifold is referred to as $k$-primitive if the star of each internal $k$-dimensional cell has $d-k+1 d$-cells (some authors call 0-primitive decompositions simple; our terminology goes back to Voronoi [44]). For decompositions of $\mathbb{R}^{d}$ by convex polyhedra, $d-k+1$ is the minimum possible number of tiles in the star of a $k$-face. If a PL-realization of a homology sphere $\mathcal{S}^{d}$ can be lifted onto a convex polytope in $\mathbb{R}^{d+1}$, then 0 -primitive vertices of $\mathcal{S}^{d}$ correspond to simple vertices of this convex polytope. When a $k$-primitive cell-decomposition of $\mathcal{M}^{d}$ is assumed to be fixed, we refer to this $k$-primitive decomposition of $\mathcal{M}^{d}$ as $k$-primitive manifold $\mathcal{M}^{d}$. The notion of $k$-primitive decomposition naturally arises in studies of space-fillers, lattice polytopes and stereohedra [44], [51], [19], [17]. For example, the affine equivalence between space-fillers and Dirichlet domains of lattices was proved by Voronoi only for 0-primitive (simple) tilings. Later Zhitomirski [51] proved that this equivalence holds for $(d-2)$-primitive tilings, and Erdahl [23] for zonotopes, but it still remains unknown whether there are more general sufficient combinatorial conditions on a space filler to be the Dirichlet domain of a lattice (for details see [17] and [19]). The existence of a lattice Dirichlet domain which is affinely isomorphic to a space-filler $\Pi$ is equivalent to the existence of a convex lifting with some special symmetries for the face-to-face lattice tiling by $\Pi[44]$.

\section{Stresses on Cell-Complexes}

If $(V, E)$ are the vertices and edges of a framework in $\mathbb{R}^{d}$, then a self-stress (or simply stress) is an assignment of real numbers $s_{i j}=s_{j i}$ to the edges, a tension if the sign is positive or a compression if the sign is negative, so that the equilibrium conditions 
$\sum_{j} s_{i j}\left(\vec{v}_{j}-\vec{v}_{i}\right)=0$ hold at each vertex $\vec{v}_{i}$. The notion of stress can be naturally generalized to $k$-stresses on cell-complexes. This generalization proves to be useful in the theory of space-fillers, the combinatorics and geometry of piecewise-linear manifolds, and the rigidity theory.

Consider a PL-realization $K^{d}$ in $\mathbb{R}^{N}$ of a cell-complex $\mathcal{K}^{d}$, and let $\vec{n}\left(C^{k-2}, C^{k-1}\right)$ be the inner unit normal to a $(k-1)$-cell $C^{k-1}$ at its $(k-2)$-face $C^{k-2}$.

Definition 3.1. A real-valued function $s(\cdot)$ on $(k-1)$-cells of $K^{d}$ is a $k$-stress if, for each internal $(k-2)$-cell $C^{k-2}$ of $K^{d}$,

$$
\sum_{C^{k-1}} s\left(C^{k-1}\right) \vec{n}\left(C^{k-2}, C^{k-1}\right)=0,
$$

where $C^{k-1}$ ranges over the $(k-1)$-cells such that $C^{k-2} \subset \partial C^{k-1}$. The quantities $s\left(C^{k-1}\right)$ are the coefficients of $k$-stresses, a tension if the sign is positive and a compression if the sign is negative.

Notice that this definition works for cell-complexes in $\mathbb{S}^{N}$ too. If not all $s\left(C^{k-1}\right)$ are zero, the $k$-stress $s$ is called non-trivial. Figure 2 illustrates the geometry of the equilibrium condition from Definition 3.1 for a 3 -stress on the star of an edge of a cell-complex in $\mathbb{R}^{3}$.

By the relationship between self-stresses in a planar framework and liftings to $\mathbb{R}^{3}$ of the cell-decomposition induced by this framework (this was originally discovered by Maxwell: see [28], [29], [35], and [36]), $d$-stresses in $d$-dimensional manifolds can be thought of as a generalization of self-stress in frameworks. To complete the analogy with stresses on frameworks the coefficients assigned to faces should be divided by their volumes, but for our purposes this would be inconvenient since our formulas would then become more cumbersome, and because we admit cells of infinite volume.

Our generalization of the notion of stress is geometrical and does not involve coordinates, whereas that of Lee [26] is algebraic and more restrictive (for finite simplicial

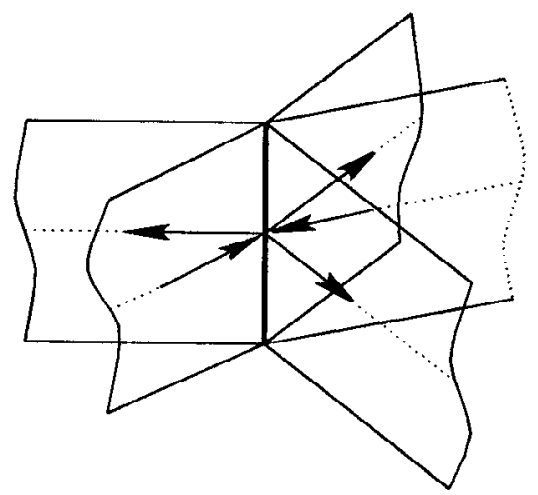

Fig. 2. Equilibrium of forces for a 3-stress. 
complexes). If $b_{a}\left(C^{k-1}\right)$ denotes Lee affine $k$-stress on a $(k-1)$-simplex $C^{k-1}$, then $s\left(C^{k-1}\right)=b_{a}\left(C^{k-1}\right) \operatorname{vol}\left(C^{k-1}\right)$. It is more natural from the geometrical point of view to assign coefficients of $k$-stresses to $k$-cells, rather than to $(k-1)$-cells, as is done in our definition, but this shifted notation was already used by several authors [26], [42], [14] and we keep to this convention. Tay et al. remarked in [42] that the notion of linear stress can be extended to general cell-complexes. Note that our notion of stress corresponds to a certain subspace of the space of linear stresses mentioned by Tay and coauthors. McMullen also uses the language of normals in [33], where he defines weights (on simple polytopes), a notion dual to stresses.

It easy to see that $k$-stresses form a linear space, and that $k$-tensions (where all coefficients of $k$-stresses are positive) and $k$-compressions (where all coefficients of $k$ stresses are negative) form congruent polyhedral cones in this linear space. We denote the space of all $k$-stresses on $M^{d}$ by $\operatorname{Stress}_{k}\left(M^{d}\right)$, and the cone of all $k$-tensions in this space by Tension $_{k}\left(M^{d}\right)$.

Theorem 3.2. Let $M^{d}$ be a PL-realization of an orientable manifold $\mathcal{M}^{d}$ in $\mathbb{R}^{d}$. For each $k=1, \ldots, d-1$, there is a polynomial mapping of degree $d-k+1$ from the space Stress $_{d}\left(M^{d}\right)$ to the space $\operatorname{Stress}_{k}\left(M^{d}\right)$, such that a d-tension (compression) is mapped to a k-tension (compression).

The proof of this theorem is based on the construction of a local Euclidean reciprocal (see Section 6) for the star $S t(v)$ of each vertex of $M^{d}$. Then define a function (polynomial in the linear parameters of the reciprocal) on all subcomplexes of this reciprocal corresponding to the stars of faces, and show that for each $(k-2)$-face of $S t(v)$ this function represents a $k$-stress which is well defined on all the manifolds. We conjecture that our mapping somehow generalizes a mapping constructed with algebraic methods by Lee in Theorem 6 of [26]. We will explore the connection between stresses on skeletons of different dimensions in a subsequent paper [24].

\section{Quality Transfer}

In this section we introduce the notion of quality transfer which allows us to make a connection between stresses and the geometry of PL-manifolds. Let $Q$ be a set of qualities and let $\mathfrak{G}$ be a group acting on $Q$. Here we consider the problem of assigning an element of $Q$, a quality, to each of the $d$-cells of $M^{d}$ so that qualities assigned to adjacent cells are governed by rules associated with the common facet. If an arbitrary quality is assigned to some $d$-cell $C_{0}$, a quality can be assigned to any other $d$-cell $C_{k}$ by translating qualities along a path connecting $C_{0}$ to $C_{k}$, using the rules associated with facets. Suppose that $f$ maps every ordered pair of adjacent cells into a group element, so that the reversed pair is mapped into the reciprocal group element.If $\mathfrak{p}=\left[C_{0}, \ldots, C_{k}\right]$ is a combinatorial path and $q$ is the quality assigned to $C_{0}$, then the quality assigned to $C_{k}$ via $\mathfrak{p}$ is given by the formula

$$
q \circ \mathfrak{f}(\mathfrak{p})=q \circ \mathfrak{f}\left(\left[C_{0}, C_{1}\right]\right) \cdot \mathfrak{f}\left(\left[C_{1}, C_{2}\right]\right) \cdots \mathfrak{f}\left(\left[C_{k-1}, C_{k}\right]\right) .
$$


The qualities assigned to cells are well defined (i.e. independent of path) if and only if every circuit lies in the kernel of $\mathfrak{f}$.

Consider a 1-complex (graph) $\mathcal{G}$ where the vertices are $d$-cells, and the edges are the $(d-1)$-cells of $\mathcal{M}^{d}$ (therefore $\mathcal{G}$ may be infinite). Two vertices share an edge if, and only if, the corresponding $d$-cells are adjacent. The graph $\mathcal{G}$ is called the combinatorial dual of $\mathcal{M}^{d}$, and it is somewhat easier to consider quality transfer on $\mathcal{G}$ rather than on $\mathcal{M}^{d}$. In this model the edges are associated with elements of $\mathfrak{G}$, and qualities are assigned to the vertices. Assigning qualities to the vertices of $\mathcal{G}$ is well defined if it is well defined over all cycles of $\mathcal{G}$. A more manageable criterion that the qualities assigned to $d$-cells are well defined is the following:

Lemma 4.1. Let $\left\{c_{i}\right\}$ be a generating system for $H_{1}\left(\mathcal{G}, \mathbb{Z}_{2}\right)$. Then quality transfer is well defined on $\mathcal{G}$ if and only if it is well defined over all cycles from $\left\{c_{i}\right\}$.

Proof. Fix a quality for a vertex $v_{0}$ of $\mathcal{G}$, and denote by $Q\left(v_{i}\right)$ the set of qualities that can be assigned to a vertex $v_{i}$ by translating qualities from $v_{0}$. Consider the 1-complex cov $\mathcal{G}$, where the vertices are the pairs $\left(v_{i}, q\left(v_{i}\right)\right)$ where $v_{i}$ is a vertex of $\mathcal{G}$ and $q\left(v_{i}\right) \in Q\left(v_{i}\right)$. Vertices $\left(v_{i}, q\right)$ and $\left(v_{j}, q^{\prime}\right)$ of cov $\mathcal{G}$ share an edge in cov $\mathcal{G}$ if and only if $v_{i}$ and $v_{j}$ are adjacent in $\mathcal{G}$ and there is $g \in \mathfrak{G}\left(\mathfrak{G}\right.$ is the group acting on $Q$ ) such that $g(q)=q^{\prime}$. It is easy to see that $\operatorname{cov} \mathcal{G}$ is a covering of $\mathcal{G}$. Since $\mathcal{G}$ is a 1-complex and $\left\{c_{i}\right\}$ is a generating system of $H_{1}\left(\mathcal{G}, \mathbb{Z}_{2}\right)$, then $\left\{c_{i}\right\}$ is also a generating system for the fundamental group $\pi(\mathcal{G})$. The covering map $p$ from $\operatorname{cov} \mathcal{G}$ onto $\mathcal{G}$ induces a monomorphism $p^{*}: \pi(\operatorname{cov} \mathcal{G}) \rightarrow \pi(\mathcal{G})$. Every cycle from $\left\{c_{i}\right\}$ is lifted onto $\operatorname{cov} \mathcal{G}$ in the trivial way (i.e. the lifting of $c_{i}$ is a single cover of $\left.c_{i}\right)$. Since $\left\{c_{i}\right\}$ is a generating system for $\pi(\mathcal{G}), p^{*}$ is an epimorphism. Therefore, $p^{*}$ is an isomorphism, and the covering map $p$ is one-to-one. As the covering is trivial, the quality transfer is well defined on $\mathcal{G}$.

For the remaining portion of this paper we shift our attention from general cellcomplexes to $d$-dimensional homology manifolds realized in $\mathbb{R}^{d}$ or $\mathbb{R}^{d+1}$. PL-manifolds with non-compact cells are permitted, if in the PL-realization non-compact cells can be retracted onto (new) compact cells (which are subsets of old non-compact cells) which span the same affine subspaces as corresponding non-compact cells. For instance, it allows the inclusion of decompositions of $\mathbb{R}^{d}$ by convex polyhedra (for example, weighted Voronoi diagrams) into the class of manifolds where the theory of $d$-stresses and liftings works. We consider all homologies with coefficients in the group of two elements: $\mathbb{Z}_{2}=\mathbb{Z} / 2 \mathbb{Z}$.

Lemma 4.2. Let $H_{1}\left(\mathcal{M}^{d}, \mathbb{Z}_{2}\right)=0$. If quality transfer is well defined over the links of all $(d-2)$-cells, then it is well defined on $\mathcal{M}^{d}$.

Proof. Since $\mathcal{M}^{d}$ is a homology manifold, one can consider a cell-decomposition $\mathcal{D}$ of $\mathcal{M}^{d}$ (in the combinatorial sense) which is dual to the original (for a description of this construction see [34] and [27]). By the definition of a polyhedral cell-complex, the cells of the decomposition of $\mathcal{M}^{d}$ can be triangulated so that all cells of the original decomposition become triangulated in a baricentrical fashion. A $k$-cell of the dual decomposition is defined as the union of the $k$-simplexes that share a common vertex in 
the baricentrical triangulation (which is the same for both the original and the dual). If $M^{d}$ has non-compact cells, we understand $\mathcal{D}$ to be a cell-decomposition of a manifold (with boundary) obtained from $M^{d}$ by contraction of all its non-compact cells onto their compact subsets (see above). This operation does not change the homotopical class and therefore preserves the homology too. Since $H_{1}\left(\mathcal{M}^{d}, \mathbb{Z}_{2}\right)=0, S k^{2}(\mathcal{D})$ has trivial $H_{1}\left(S k^{2}(\mathcal{D}), \mathbb{Z}_{2}\right)$. In other words, a cycle on $S k^{1}(\mathcal{D})$ can be represented as the sum of the boundaries of 2-cells of $\mathcal{D}$. It is easy to see that the dual graph $\mathcal{G}\left(\mathcal{M}^{d}\right)$ is a subgraph of $S k^{1}(\mathcal{D})$ and coincides with $S k^{1}(\mathcal{D})$ when $\mathcal{M}^{d}$ is a closed manifold. The $d$-cells having facets that belong to $\partial \mathcal{M}^{d}$ require a more detailed consideration. If $\mathcal{M}^{d}$ has a boundary, $S k^{2}(\mathcal{D})$ has edges and vertices corresponding to boundary $(d-1)$-cells, and 2-cells corresponding to boundary $(d-2)$-cells. Indeed, any cycle of $\mathcal{G} \subset S k^{1}(\mathcal{D})$ is the boundary of a chain whose carrier does not contain such 2-cells. Thus, the cycles of $\mathcal{G}$ that correspond to the stars of internal $(d-2)$-dimensional cells form a generating system for $H^{1}\left(\mathcal{G}, \mathbb{Z}_{2}\right)$. By Lemma 4.1 , quality transfer is well defined on $\mathcal{M}^{d}$.

The technique of quality transfer on Euclidean tilings goes back to Voronoi [44]. However, his technique was essentially homotopical, not homological (see [19] and [35]). The homological version of this procedure was later implicitly used by several authors, including Crapo, Whiteley [14], [48] and McMullen [31].

If $M^{d}$ is a fixed realization in $\mathbb{R}^{d}$ of a manifold $\mathcal{M}^{d}$, one can attempt to assign +1 or -1 to each cell (recall that every $d$-cell is embedded into $\mathbb{R}^{d}$ ) so that two adjacent $d$-cells have the same orientation if and only if their outer normals at their common facet have opposite directions. By the definition of a cell-complex, $\mathcal{M}^{d}$ can be baricentrically triangulated. One can prove that $\mathcal{M}^{d}$ is orientable in the sense defined above, if and only if it is orientable as a simplicial complex (in the usual sense). Since we need our geometrical notion of orientation in the following section, we now show that any manifold $\mathcal{M}^{d}$ with $H_{1}\left(\mathcal{M}^{d}, \mathbb{Z}_{2}\right)=0$ is orientable in this sense.

Assuming that for a $d$-cell $C_{0}$ of $M^{d}$ the orientation of the embedding is $o$, we define the orientation for an adjacent cell $C_{1}$ as follows. If outer normals to $C_{1}$ and $C_{0}$ at their common facet have opposite directions (when $C_{1}$ and $C_{0}$ are convex, it means that they point to different half-spaces determined by the facet), assign orientation $+o$ to $C_{1}$; if the normals are co-oriented, assign orientation $-o$ to $C_{1}$. Since $\mathcal{M}^{d}$ is strongly connected, an orientation can be assigned to any cell of $M^{d}$ by transferring the orientation along paths of adjacent cells in this fashion. We have to show that the orientation assigned to a cell in this manner is independent of a particular path used for the transfer, and is therefore well defined. By Lemma 4.2, this requires that the transfer of orientation be well defined over the links of the internal $(d-2)$-cells which are all $\mathbb{S}^{1}$. Since the closure of each cell of $\mathcal{M}^{d}$ is embedded into $\mathbb{R}^{d}$, the orientation is correctly defined over the links of all $(d-2)$-cells, so, by Lemma 4.2 , the orientation can be properly introduced on all of $M^{d}$. Throughout the paper we denote the orientation of a cell $C$ by $o(C)$.

\section{Stresses, Splines and Liftings}

Let $K^{d}$ be a PL-realizationof a combinatorial cell-complex $\mathcal{K}^{d}$ in $\mathbb{R}^{d}$. A family of affine functions $L\left(\mathbf{x} ; C^{d}\right)$ on $\mathbb{R}^{d}$ corresponding to the $d$-cells of $K^{d}$ is referred to as a lifting of $K^{d}$ if $L\left(\mathbf{x} ; C^{d}\right)$ determines a PL-realization $L^{d}$ of $\mathcal{K}^{d}$ in $\mathbb{R}^{d+1}$. In other words, each 
$d$-cell of $K^{d}$ is the vertical projection of the corresponding $d$-cell of the realization $L^{d}$ determined by the affine functions $L\left(\mathbf{x} ; C^{d}\right)$. Since $L^{d}$ is a PL-realization of $\mathcal{K}^{d}$, each $k$ cell $(0 \leq k \leq d)$ of $K^{d}$ is a vertical projection of the corresponding $k$-cell of $L^{d}$. A lifting is called sharp if each pair of adjacent $d$-cells of $K^{d}$ is lifted onto distinct hyperplanes in $\mathbb{R}^{d+1} . L\left(\mathbf{x} ; C^{d}\right)$ is called locally convex (concave) if on each sub-complex of $K^{d}$ which is embedded into $\mathbb{R}^{d}, L\left(\mathbf{x} ; C^{d}\right)$ is a convex (concave) PL-function. We refer to the angle between two adjacent $d$-cells of a lifting as a dihedral angle. Denote the linear space of liftings defined up to the choice of an affine function by $\operatorname{Lift}\left(K^{d}\right)$. Locally convex liftings form the polyhedral cone $C \operatorname{Lift}\left(K^{d}\right)$ in this space. Lifting is a natural generalization of the notion of continuous PL-function $\left(C_{1}^{0}\right.$-spline) on a cell-decomposition of a polyhedral region. A convex lifting is a convex PL-function on the region. The conception of lifting is very convenient in studies of stresses in planar frameworks, and in the analysis of polyhedral scenes [2], [12], [14], [45], [47].

Let $M^{d}$ be a PL-realization in $\mathbb{R}^{d}$ of a manifold $\mathcal{M}^{d}$. A real-valued function $c_{1}^{0}$ on the ordered pairs of adjacent $d$-cells of $M^{d}$ is called a $C_{1}^{0}$-cofactor if

1. $c_{1}^{0}\left(\left[C, C^{\prime}\right]\right)=-c_{1}^{0}\left(\left[C^{\prime}, C\right]\right)$,

2. for each internal $(d-2)$-cell $C^{d-2}$,

$$
\sum_{i=1}^{i=n} c_{1}^{0}\left(\left[C_{i}, C_{i+1}\right]\right) \vec{n}\left(\left[C_{i}, C_{i+1}\right]\right)=0
$$

where $\left[C_{1}, \ldots, C_{n}, C_{n+1}=C_{1}\right]$ is a cyclical order of the $d$-cells making contact in $C^{d-2}$, and $\vec{n}\left(\left[C_{i}, C_{i+1}\right]\right)$ is the outer unit normal to $C_{i}$ at its facet shared with $C_{i+1}$. We also use the term $C_{1}^{0}$-cofactor for referring to the value of a function $c_{1}^{0}$ ( ) on a facet.

A lifting $L(\mathbf{x} ; C)=\langle\vec{a}(C), \mathbf{x}\rangle+a_{0}(C)$ determines a $C_{1}^{0}$-cofactor $c_{1}^{0}$ by $c_{1}^{0}\left(\left[C, C^{\prime}\right]\right)=$ $\left.\left\langle\vec{a}\left(C^{\prime}\right)-\vec{a}(C)\right), \vec{n}\left(\left[C, C^{\prime}\right]\right)\right\rangle$. In Theorem 5.1 we show that if $H_{1}\left(\mathcal{M}^{d}, \mathbb{Z}_{2}\right)=0$, then a cofactor determines a lifting up to the choice of an affine function. The following theorem connects stresses and liftings for PL-realizations of a manifold with $H_{1}=0$ over $\mathbb{Z}_{2}$.

Theorem 5.1. Let $M^{d}$ be a PL-realization of a manifold $\mathcal{M}^{d}$ with trivial $H_{1}\left(\mathcal{M}^{d}, \mathbb{Z}_{2}\right)$. The linear space Stress ${ }_{d}\left(M^{d}\right)$ is isomorphic to the linear space Lift $\left(M^{d}\right)$. All-non-zero stresses correspond to sharp liftings. If $M^{d}$ is an embedding, then Tension ${ }_{d}\left(M^{d}\right) \cong$ $\operatorname{CLift}\left(M^{d}\right)$.

Proof. Take the set $A$ of affine scalar-valued functions on $\mathbb{R}^{d}$ to be the set of qualities. This set has a linear space structure and acts on itself by addition. We denote this transferal group by $\mathfrak{G}$. If each ordered pair of adjacent cells is associated with a group element from $\mathfrak{G}$, and an affine function is assigned to any particular $d$-cell, affine functions can be assigned to adjacent cells using the action of $\mathfrak{G}$ on $A$. Lemma 4.2 gives sufficient conditions that the quality assigned to a cell in this manner be independent of the path used for the transfer, and is therefore well defined. As an application of this lemma, we shall establish a linear correspondence between $d$-stresses and liftings.

Let $s$ be a non-trivial $d$-stress on $M^{d}$, and fix the orientations of the $d$-cells of $M^{d}$ so that the orientations agree for pairs of adjacent cells as was described above. If $d$-cells 
$C_{1}, C_{2}$ are adjacent, denote by $E_{1,2}(\mathbf{x})=\langle\vec{n}, \mathbf{x}\rangle-c=0$ the equation of their common facet $C^{d-1}$ which is adjusted so that the vector $\vec{n}$ is an outer unit normal to $C_{1}$ at $C^{d-1}$ (if $C_{1}$ is convex, this is the same as saying that $E_{1,2}(\mathbf{x}) \leq 0$ on $\left.C_{1}\right)$. If $s\left(C_{1}, C_{2}\right)=s\left(C_{2}, C_{1}\right)$ is the stress on $C^{d-1}$, let $g\left(\mathbf{x},\left[C_{1}, C_{2}\right]\right)=s\left(C_{1}, C_{2}\right) o\left(C_{1}\right)(\langle\vec{n}, \mathbf{x}\rangle-c)$ be the affine function associated with the ordered pair $\left[C_{1}, C_{2}\right]$ (as an element of $\mathfrak{G}$ ). If $q\left(\mathbf{x}, C_{1}\right)$ is the affine function for $C_{1}$, then the affine function for $C_{2}$ is given by $q\left(\mathbf{x}, C_{1}\right)+g\left(\mathbf{x},\left[C_{1}, C_{2}\right]\right)$. It is easy to see that this definition is symmetric and for the path $\left[C_{1}, C_{2}, C_{1}\right]$ we have $g\left(\mathbf{x},\left[C_{1}, C_{2}\right]\right)+g\left(\mathbf{x},\left[C_{2}, C_{1}\right]\right)=0$. Since $g(\mathbf{x}, \mathfrak{p})$ is defined on two-element paths, it is also defined on arbitrary paths. Since $\mathcal{M}^{d}$ is a manifold, the link of each internal $(d-2)$-cell is $\mathbb{S}^{1}$. Consider an ordered circuit $\left[C_{1}, \ldots, C_{n}, C_{n+1}\right]$, where $C_{i}$ are the $d$-cells from the star of an internal $(d-2)$-cell $C^{d-2}$ and $C_{n+1}=C_{1}$. It is easy to see that $g\left(\mathbf{x},\left[C_{1}, \ldots, C_{n}, C_{1}\right]\right)=0$ if and only if

$$
\vec{v}=\sum_{i=1}^{i=n} s\left(\left[C_{i}, C_{i+1}\right]\right) o\left(C_{i}\right) \vec{n}\left(\left[C_{i}, C_{i+1}\right]\right)=0
$$

Rotate $\vec{v}$ by $90^{\circ}$ in the orthogonal complement to span $\left(C^{d-2}\right)$ in such way that the result of the rotation of $\left(o\left(C_{1}\right) \vec{n}\left(\left[C_{1}, C_{2}\right]\right)\right)$ becomes the inner unit normal to the common facet of $C_{1}$ and $C_{2}$ at $C^{d-2}$. Denote this rotation operator by $r$. Consider instead of $S t\left(C^{d-2}\right)$ the section of $S t\left(C^{d-2}\right)$ with a perpendicular 2-plane. By convention we refer to the direction of rotation from $C_{1}$ to $C_{2}$ through their common facet as clockwise. For each $C_{i}, i=1, \ldots, n$, call the facet where $C_{i}$ contacts $C_{i-1}\left(C_{n}\right.$ if $\left.i=1\right)$ the first facet and the facet where $C_{i}$ contacts $C_{i+1}$ the second facet. If $o\left(C_{i}\right)=o\left(C_{1}\right)$, then the direction of rotation from the first facet of $C_{i}$ to the second is clockwise. In this case, the rotation transforms vector $o\left(C_{1}\right) \vec{n}\left(\left[C_{i}, C_{i+1}\right]\right)$ into the inner unit normal to the common facet of $C_{i}$ and $C_{i+1}$ at $C^{d-2}$. If $o\left(C_{i}\right)=-o\left(C_{1}\right)$, then the direction of rotation from the first facet of $C_{i}$ to the second is counter-clockwise. It is easy to see that, in this case, $o\left(C_{1}\right) \vec{n}\left(\left[C_{i}, C_{i+1}\right]\right)$ is also transformed into the inner unit normal to the common facet of $C_{i}$ and $C_{i+1}$ at $C^{d-2}$. Thus $r(\vec{v})=0$, for $r(\vec{v})$ is the vector sum of $d$-stresses at $C^{d-2}$. It therefore follows that formula (1) holds.

By Lemma 4.2, since the quality transfer is well defined over the links of all $(d-2)$ cells, it is well defined on all of $\mathcal{M}^{d}$. Our construction provides continuous gluing over facets of $M^{d}$. If an affine function is fixed for any $d$-cell (the "origin"), affine functions can be assigned to the other cells using the group $\mathfrak{G}$ and the quality transfer. The resulting family of affine functions is a lifting by construction and Lemma 4.2. It is easy to see that if the coefficients of $d$-stresses are all-non-zero, then the lifting is sharp.

Conversely, let $L^{d}=L(\mathbf{x}, C)$ be a lifting of $M^{d}$. If $L(C)$ is the lifting of a $d$-cell $C$ of $M^{d}$, define $o(L(C)):=o(C)$ (recall that the orientations of embedding $o(C)$ have been adjusted for the $d$-cells of $M^{d}$ ). The $d$-stress on $M^{d}$ corresponding to $L^{d}$ can be found in the following way. Let $L\left(C_{1}\right)$ and $L\left(C_{2}\right)$ be adjacent $d$-dimensional cells of the lifting projecting onto cells $C_{1}$ and $C_{2}$ of the "flat" realization $M^{d}$, and defined by affine functions $L\left(\mathbf{x}, C_{1}\right)=\left\langle\vec{a}_{1}, \mathbf{x}\right\rangle+c_{1}$ and $L\left(\mathbf{x}, C_{2}\right)=\left\langle\vec{a}_{2}, \mathbf{x}\right\rangle+c_{2}$. Let $\vec{n}_{1}$ be the outer unit normal to $C_{1}$ at facet $C^{d-1}$ shared with $C_{2}$. Now, we can assign the coefficient of stress $o\left(L\left(C_{1}\right)\right)\left\langle\vec{a}_{2}-\vec{a}_{1}, \vec{n}_{1}\right\rangle$ to the facet $C^{d-1}$. Reversing the arguments in the proof of formula (1) from the first part of the theorem, one can see that introduced quantities 


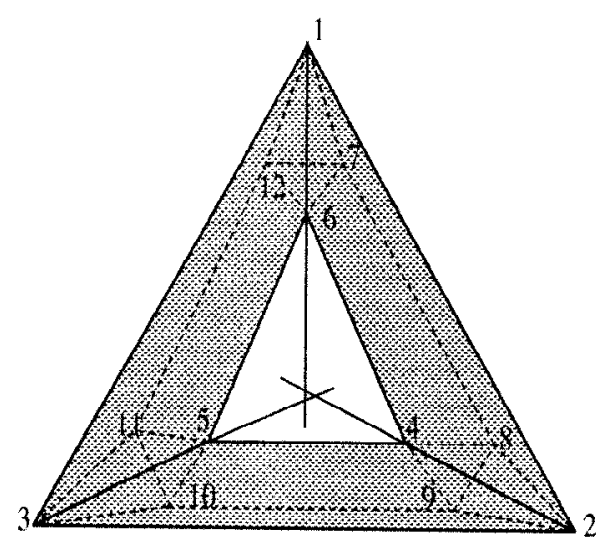

Fig. 3

$o\left(L\left(C_{1}\right)\right)\left\langle\vec{a}_{2}-\vec{a}_{1}, \vec{n}_{1}\right\rangle$ are actually coefficients of $d$-stresses. Notice that these arguments have local character and do not employ the condition that $H_{1}\left(\mathcal{M}^{d}, \mathbb{Z}_{2}\right)=0$.

Since, in the second part of Theorem 5.1, the homological condition that $H_{1}\left(\mathcal{M}^{d}, \mathbb{Z}_{2}\right)=$ 0 is not used, this theorem constructs a monomorphism from $\operatorname{Lift}\left(M^{d}\right)$ into $\operatorname{Stress}_{d}\left(M^{d}\right)$ for an arbitrary orientable $\mathcal{M}^{d}$. This monomorphism maps a $C_{1}^{0}$-cofactor $c_{1}^{0}$ to a $d$-stress $s$ via formula $s\left(C^{d-1}\right)=c_{1}^{0}\left(C^{d-1}\right)$, where $C^{d-1}$ is an internal facet of $M^{d}$. Notice that in the proof we did not assume the convexity of cells. Theorem 5.1 was independently proved by Whiteley for homology 3-spheres in preprint [14], although the topological part of the proof was only sketched and the condition $H_{1}\left(\mathcal{M}^{d}, \mathbb{Z}_{2}\right)=0$ was not mentioned as sufficient for the existence of the isomorphism between $\operatorname{Stress}_{d}\left(M^{d}\right)$ and $\operatorname{Lift}\left(M^{d}\right)$.

Although the space of lifting is always a subspace of the space of stress, the converse is not necessarily true. A realization of the torus depicted in Fig. 3 demonstrates that in general one cannot drop the condition $H_{1}\left(\mathcal{M}^{d}, \mathbb{Z}_{2}\right)=0$ (triangles (123), (456) are not loaded; edges (16), (24), (35) have arbitrary tensions, which are resolved by other edges; since lines 16, 24, 35 do not pass through a common point, the torus does not lift).

If $\Delta$ is a cell-decomposition of a region in $\mathbb{R}^{d}$, then a lifting of $\Delta$ is a continuous PL-function on $\Delta$, i.e. a $C_{1}^{0}$-spline. More generally, a $C_{m}^{r}$-spline over $\Delta$ is a $C^{r}$-smooth function which is represented by polynomials of degree at most $m$ on each $d$-cell. Such functions form a vector space over $\mathbb{R}$, which is denoted by $S_{m}^{r}(\Delta)$. The theory of splines on manifolds embedded into $\mathbb{R}^{d}$ is similar to the theory of stresses and liftings. Recall that coefficients of $d$-stresses define gluing of affine functions over facets of a decomposition in the way shown in Section 5. Regarding smooth splines as generalizations of PLfunctions, one can say that $C_{r}^{r-1}$-cofactors play the same role for $C_{r}^{r-1}$-splines as stresses for $C_{1}^{0}$-splines. They define the smooth gluing of polynomial patches over facets (the core of the theory of cofactors of smooth splines can be found in [50].) Let us fix orientations for the links of all internal $(d-2)$-cells. The orientation of the link of a $(d-2)$-cell 
induces the orientations of normals to supporting hyperplanes of facets making contact in this $(d-2)$-cell.

Definition 5.2. A real-valued function $c_{r}^{r-1}()$ on the $(d-1)$-cells of $\Delta$ is called a $C_{r}^{r-1}$-cofactor if, for each internal $(d-2)$-cell $C^{d-2}$ of $\Delta$, and for each non-negative integer vector $\left(i_{1}, \ldots, i_{n}\right)$ such that $i_{1}+\cdots+i_{n}=r$,

$$
\sum_{C^{d-2} \subset \partial F} c_{r}^{r-1}(F) n_{1}^{i_{1}}(F) \cdots n_{d}^{i_{d}}(F)=0
$$

where $F$ ranges over all facets making contact at $C^{d-2}$ and $n_{1}(F), \ldots, n_{d}(F)$ are the coordinates of the unit normal to the hyperplane spanned by $F$ whose orientation is induced by the fixed orientation of the link of $C^{d-2}$.

Clearly, the $C_{r}^{r-1}$-cofactors form a linear space, which we denote by $\operatorname{COF}_{r}^{r-1}(\Delta)$. The following lemma [50] explains the importance of the above definition.

Lemma 5.3. Let $c_{r}^{r-1}$ be a $C_{r}^{r-1}$-cofactor for $\Delta$. Then, adopting the notation from Definition 5.2, for every internal $(d-2)$-cell $C^{d-2}$ of $\Delta$ we have

$$
\sum_{C^{d-2} \subset \partial F} c_{r}^{r-1}(F)\left(n_{1}(F) x^{1}+\cdots+n_{d}(F) x^{d}+n_{d+1}(F)\right)^{r}=0,
$$

where $F$ ranges over all facets making contact at $C^{d-2},\left(x_{1}, \ldots, x_{d}\right)$ are the usual Euclidean coordinates in $\mathbb{R}^{d}$ and $n_{1}(F) x_{1}+\cdots+n_{d}(F) x_{d}+n_{d+1}(F)=0$ is an equation for $F$.

This statement is known as the $C_{r}^{r-1}$-cofactor reduction lemma [50]. The following lemma explains why $C_{r}^{r-1}$-cofactors determine the smooth gluing of polynomial patches (for the proof see [8]).

Lemma 5.4. Let function $f$ be represented by polynomials of at most degree $m$ on each $d$-cell of $\Delta$. Then $f$ is a $C_{m}^{r}$-spline if and only if for each pair of adjacent $d$ cells the difference between polynomials corresponding to these cells is divided by the $(r+1)$-power of an affine function vanishing on the common facet of these $d$-cells.

The following theorem, which generalizes Billera's theorem [9], [50] for $d>2$ is a consequence of Lemma 4.2 on quality transfer. It underlines the analogies between stresses and cofactors of $C_{r}^{r-1}$-splines.

Theorem 5.5. If $H_{1}\left(\Delta, \mathbb{Z}_{2}\right)=0$, then

$$
\operatorname{dim} S_{r}^{r-1}(\Delta)=\left(\begin{array}{c}
r+d \\
d
\end{array}\right)+\operatorname{dim} \operatorname{COF}_{r}^{r-1}(\Delta) .
$$

Proof. A proof of this theorem can be obtained by direct substitution of $C_{1}^{0}$-cofactors by $C_{r}^{r-1}$-cofactors in the proof of Theorem 5.1. 


\section{Reciprocals, Liftings and Stresses}

Consider a planar framework $(V, E)$ that is in a state of static equilibrium, and assume that the framework determines a cell-decomposition $D(V, E)$ of $\mathbb{R}^{2}$ (assuming that the framework has vertices at infinity), or of a simply connected region in $\mathbb{R}^{2}$. Consider a vertex of $(V, E)$. The sum of vectors of stresses applied to this vertex is equal to zero. Therefore, when rotated on $90^{\circ}$ clockwise they form a polygon (self-intersecting in general). It was noticed by Maxwell (for a proof see [45]) that the positions of rotated edges of $(V, E)$ can be adjusted so that they form a reciprocal graph (or simply reciprocal). Each edge of this reciprocal corresponds to an edge of $(V, E)$ and each vertex to a cell of $D(V, E)$ (one vertex corresponds to the complement of $D(V, E)$ if any). We introduce and explore a similar notion for $d$-manifolds in $\mathbb{R}^{d}$ (see also [48] and [14]).

The combinatorial dual graph $\mathcal{G}\left(\mathcal{M}^{d}\right)$ of a manifold $\mathcal{M}^{d}$ is a (multi)graph where the vertices are the $d$-cells of $\mathcal{M}^{d}$, and the edges are the internal $(d-1)$-cells of $\mathcal{M}^{d}$.

A reciprocal of a PL-realization $M^{d}$ of a manifold $\mathcal{M}^{d}$ in $\mathbb{R}^{d}$ is a rectilinear realization $R$ in $\mathbb{R}^{d}$ of the combinatorial dual graph $\mathcal{G}\left(\mathcal{M}^{d}\right)$ such that the edges of $R$ are perpendicular to the corresponding facets. If none of the edges of a reciprocal collapses into a point, then the reciprocal is called non-degenerate.

Let $v\left(C_{1}\right)$ and $v\left(C_{2}\right)$ be vertices of a reciprocal $R$ corresponding to adjacent $d$-cells $C_{1}$ and $C_{2}$. If $\vec{v}\left(C_{2}\right)-\vec{v}\left(C_{1}\right)$ is co-oriented with an outer normal to $C_{1}$ at the facet shared with $C_{2}$, then the edge $\left[v\left(C_{2}\right) v\left(C_{1}\right)\right]$ is called properly oriented. Otherwise it is called improperly oriented. If realization $M^{d}$ is an embedding and all edges of $R$ are properly oriented, $R$ is called a convex reciprocal (since the cycles of $R$ corresponding to the stars of the $(d-2)$-cells are convex in this case). Reciprocals with one fixed vertex form a linear space. Denote it by $\operatorname{Rec}\left(M^{d}\right)$. Reciprocals were originally considered by Maxwell [28] in connection with stresses in plane frameworks. The linear space of planar reciprocals was studied in [12]. Convex reciprocals form a cone $\operatorname{Rec}\left(M^{d}\right)$ in the linear space $\operatorname{Rec}\left(M^{d}\right)$. Maxwell noticed that convex reciprocals corresponded to convex liftings of planar cell-complexes. The convex reciprocals were studied in [4], [12], [13], [45], [35], and [36]. The following theorem is a simple consequence of the definitions given above and of affine properties of affine spaces. It holds regardless of the homological properties of a manifold.

Theorem 6.1. Let $M^{d}$ be a PL-realization of a manifold $\mathcal{M}^{d}$ in $\mathbb{R}^{d}$. There is an isomorphism between Lift $\left(M^{d}\right)$ and Rec $\left(M^{d}\right)$. Sharp liftings correspond to non-degenerate reciprocals. If $M^{d}$ is an embedding, then convex dihedral angles correspond to properly oriented edges of the reciprocal, concave dihedral angles correspond to improperly oriented edges, and $C \operatorname{Lift}\left(M^{d}\right) \cong \operatorname{CRec}\left(M^{d}\right)$.

Proof. Let $R$ be a reciprocal for $M^{d}$, let $v\left(C_{1}\right)$ and $v\left(C_{2}\right)$ be vertices of $R$ corresponding to $d$-cells $C_{1}$ and $C_{2}$, and let $\vec{n}$ be the outer unit normal to $C_{1}$ at the facet $F$ shared with $C_{2}$. Since $\vec{v}\left(C_{2}\right)-\vec{v}\left(C_{1}\right)$ is orthogonal to $F$, there is $c \in \mathbb{R}$ such that $\left\langle\vec{v}\left(C_{2}\right)-\vec{v}\left(C_{1}\right), \mathbf{x}\right\rangle+c=0$ is the equation of $F$. A lifting corresponding to $R$ can be constructed as follows. If $L\left(\mathbf{x} ; C_{1}\right)$ is an affine function corresponding to $C_{1}$, then the affine function corresponding to $C_{2}$ is given by $L\left(\mathbf{x} ; C_{1}\right)+\left\langle\vec{v}\left(C_{2}\right)-\vec{v}\left(C_{1}\right), \mathbf{x}\right\rangle+c$. Let us fix an arbitrary affine function for a $d$-cell $C_{0}$. Connect a $d$-cell $C$ with $C_{0}$ by a combinatorial path. We determine the 
affine function $L(\mathbf{x} ; C)$ corresponding to $C$ via the use of the above formula. Since every cycle of $\mathcal{G}\left(\mathcal{M}^{d}\right)$ is realized as a rectilinear cycle of $R$ in $\mathbb{R}^{d}, L(\mathbf{x} ; C)$ is path independent and is therefore well defined. It is easy to see that $L(\mathbf{x} ; C)$ determines a PL-realization of $\mathcal{M}^{d}$ in $\mathbb{R}^{d+1}$ where non-degenerate dihedral angles correspond to non-degenerate edges of $R$.

Let $L^{d}=L(\mathbf{x} ; C)$ be a lifting of $M^{d}$, and let $L\left(\mathbf{x} ; C_{1}\right)=\left\langle\vec{a}_{1}, \mathbf{x}\right\rangle+c$ and $L\left(\mathbf{x} ; C_{2}\right)=$ $\left\langle\vec{a}_{2}, \mathbf{x}\right\rangle+c$ be the affine functions determining adjacent $d$-cells of $L^{d}$ which correspond to $d$-cells $C_{1}$ and $C_{2}$ of $M^{d}$.

If $v_{1}$ is a vertex of a reciprocal corresponding to $C_{1}$, then the vertex corresponding to $C_{2}$ is given by $v_{1}+\vec{a}_{2}-\vec{a}_{1}$. Fix a vertex corresponding to a $d$-cell $C_{0}$ at the origin and construct all other vertices and edges of the reciprocal using this formula. It is easy to see that the resulting rectilinear 1-complex is a reciprocal for $M^{d}$ and non-degenerate dihedral angles of $L^{d}$ correspond to non-degenerate edges of the reciprocal.

In the two-dimensional case, the connection between liftings and reciprocals was first noticed by Maxwell. The isomorphism between spaces of liftings and reciprocals for manifolds in $\mathbb{R}^{2}$ was proved by Crapo and Whiteley [12], [13]. The relationship between convex liftings of cell-decompositions of $\mathbb{R}^{d}$ and convex reciprocals was also shown in [3].

\section{Voronoi Diagrams: Duality, Projections and Stresses}

The aim of this section is to show some important connections between stresses, liftings and weighted Voronoi and Delaunay diagrams. A point $s$ in Euclidean space $\mathbb{R}^{d}$ is said to be additively weighted if it is associated with a real number $w(s)$ referred to as the weight of $s$. The weighted distance from $s$ is

$$
\mathbf{d}^{2}(s, x)-w(s)
$$

where $\mathbf{d}(s, x)$ denotes the Euclidean distance between points $s$ and $x$.

Let $S$ be a discrete set of additively weighted points in $\mathbb{R}^{d}$, such that all weights are bounded in absolute value. Points of $S$ are called sites. A point $x \in \mathbb{R}^{d}$ belongs to the Voronoi domain of a site $p \in S$ with weight $w(p)$ if and only if

$$
\mathbf{d}^{2}(p, x)-w(p) \leq \mathbf{d}^{2}(s, x)-w(s)
$$

for all $s \in S$.

It is easy to see that each non-empty domain of a weighted Voronoi diagram is a convex polyhedron and that these polyhedra form a face-to-face decomposition of $\mathbb{R}^{d}$. Such a decomposition is called an (additively) weighted Voronoi diagram (also often referred to as a weighted Dirichlet decomposition, or a power diagram). If all weights are equal, then the diagram is referred to as a Voronoi (Dirichlet) diagram. Let $\Delta$ be a weighted Voronoi diagram. Then $\Delta$ can be represented as a weighted Voronoi diagram in different ways. The location and the weight of at least one site can be chosen arbitrarily. (Notice that weighted sites can lie outside their domains.) Weighted Voronoi diagrams have many applications. (For more information on weighted Voronoi diagrams see [6]). 
Algorithms constructing weighted Voronoi diagrams and data structures related to these diagrams are described in [6] and [20]. A discrete set of points $S$ in $\mathbb{R}^{d}$ uniquely defines a special decomposition of the convex hull of $S$ which is combinatorially and metrically dual to the Voronoi diagram of $S$ [16], [18]. Such a decomposition is called a Delaunay decomposition. Every $d$-cell of that decomposition is inscribed into a sphere which does not contain points of $S$ in its interior.

Let $S$ be a discrete set of points in $\mathbb{R}^{d}$. A convex polytope $P$ in $\mathbb{R}^{d}$ is called a Delaunay cell of the system of points $S$ if:

1. all vertices of $P$ belong to $S$;

2. there is a sphere circumscribed around $P$;

3. no points of $S$ except the vertices of $P$ lie inside or on the sphere.

Delaunay cells form a face-to-face decomposition of conv $S$. This decomposition is defined uniquely by $S$. Delaunay decompositions have many applications in computational geometry, mesh generation, the theory of lattices, mathematical crystallography, etc. One of the generalizations of Delaunay decompositions, the weighted Delaunay decompositions, are intensively employed in computational geometry [21], [22]. For generically distributed sites and weights, the weighted Delaunay decomposition is a triangulation. Such triangulations are called regular. A sphere circumscribed around a cell of a Delaunay decomposition is called an empty sphere. Informally, a Delaunay decomposition consists of cells which sit in their own empty spheres. Generalizing Delaunay diagrams to weighted Delaunay diagrams we replace standard Euclidean spheres by virtual spheres defined through weighted distances.

Let $S$ be a discrete set of points in $\mathbb{R}^{d}$. Let $W$ be a set of real weights bounded in absolute value which are associated with these points. A polytope $P \subset \mathbb{R}^{d}$ is called a weighted Delaunay cell of the system of weighted points $(S, W)$ if:

1. all vertices of $P$ belong to $S$;

$2^{*}$. there is a point $c(P) \in \mathbb{R}^{d}$ called the weighted center of $P$ and $r(P) \in \mathbb{R}$ such that, for every vertex $v$ of $P$,

$$
\mathbf{d}^{2}(c(P), v)-w(v)=r(P)
$$

$3^{*}$. for each $s \in S$ which is not a vertex of $P$,

$$
r(P)<\mathbf{d}^{2}(c(P), s)-w(s) .
$$

Weighted Delaunay cells form a face-to-face decomposition of conv $S$.

The weighted vertices of a weighted Delaunay decomposition are the weighted sites for the dual weighted Voronoi diagram. The duality between weighted Delaunay and Voronoi decompositions can be proved exactly in the same way as the duality for ordinary Voronoi and Delaunay decompositions (see [16] and [18]). The duality between Voronoi diagrams and Delaunay decompositions can be naturally generalized in the following way.

Let $\Delta$ be an arbitrary locally finite decomposition of $\mathbb{R}^{d}$ into compact convex 
polyhedra. A decomposition $\Delta^{*}$ of $\mathbb{R}^{d}$ is called a dual of $\Delta$ if the following conditions hold:

1. combinatorial duality: there is a one-to-one correspondence between $m$-dimensional faces of $\Delta^{*}$ and $(d-m)$-dimensional faces of $\Delta, 0 \leq m \leq d$; this correspondence induces an isomorphism between the incidence graphs (infinite) of $\Delta$ and $\Delta^{*}$;

2. orthogonality and proper orientation: the 1-skeleton of $\Delta^{*}$ is a convex reciprocal for $\Delta$.

It is easy to see that this relationship is reciprocal, i.e. if $\Delta^{*}$ is a dual of $\Delta$, then $\Delta$ is a dual of $\Delta^{*}$. The above definition captures all properties of the duality between ordinary (or weighted) Voronoi and Delaunay decompositions except for the relationship between Voronoi sites and vertices and Delaunay centers and vertices. The property that Delaunay vertices are Voronoi centers and vice versa does not find its generalization in this construction. One can ask what does the existence of a dual decomposition for an arbitrary $\Delta$ imply in terms of the geometry of $\Delta$ ? In this section we show that two decomposition are dual if and only if it is possible to present one of them as a weighted Voronoi diagram $(S, W)$ and the other as the dual Delaunay decomposition (later we show that these two properties are equivalent). The notion of dual decomposition can also be regarded as a $d$-dimensional analog of the notion of planar convex reciprocal.

If $\Delta^{*}$ is a dual of $\Delta$, then any decomposition obtained from $\Delta^{*}$ by scaling and translating is also a dual of $\Delta$. It is obvious that the vector sum of any two dual decompositions of $\Delta$ is again a dual decomposition for $\Delta$. Thus all decompositions (considered up to translation) which are dual for decomposition $\Delta$ form a cone $\operatorname{Dual}(\Delta)$.

Theorem 7.1. A decomposition $\Delta$ of $\mathbb{R}^{d}$ has a dual decomposition if and only if $\Delta$ has a d-tension. The cone of dual decompositions Dual $(\Delta)$ is isomorphic to the cone of tensions Tension $_{d}(\Delta)$.

Proof. Let $\Delta^{*}$ be a dual for $\Delta$. By definition, $S k^{1}\left(\Delta^{*}\right)$ is a non-degenerate convex reciprocal for $\Delta$. Thus, by Theorem 6.1 we have a monomorphism from the cone of dual decompositions into the cone of tensions. In fact, these cones are isomorphic. The cone of tensions is isomorphic to the cone of convex liftings by the results of Section 5 . Consider a convex lifting of $\Delta$. Let $p \in \mathbb{R}^{d+1}$ be a point lying above this surface. Intersections of cones dual to the stars of vertices of the lifting with $\mathbb{R}^{d}$ are convex polyhedra which form a face-to-face decomposition of $\mathbb{R}^{d}$. This decomposition is a dual of $\Delta$ by construction.

Aurenhammer [4] proved that a finite decomposition of $\mathbb{R}^{d}$ is the projection of a convex polyhedral surface if and only if it is a weighted Voronoi diagram. This theorem holds for infinite decompositions by finite polyhedra as well (adopting the proof is straightforward). The following theorem follows from Theorems 5.1 and 6.1 and Aurenhammer's theorem.

Theorem 7.2. A decomposition of $\mathbb{R}^{d}$ by finite convex polyhedra is a weighted Voronoi decomposition if and only if it has a d-tension. 
If $\left(S_{1}, W_{1}\right)$ and $\left(S_{2}, W_{2}\right)$ are two diagrams representing a decomposition $\Delta$, then there is a collection of weights $W$, such that $\left(S_{1}+S_{2}, W\right)$ represents $\Delta$. If a weighted diagram $(S, W)$ represents a decomposition $\Delta$, and $h(S)$ is a homothetic image of $S$, then there is a set of weights $W^{\prime}$ such that $\Delta$ is a weighted diagram with site set $h(S)$ and weight set $W^{\prime}$. The same is true about translating the set of sites, i.e. if $t(S)$ is a translated copy of $S$, then there is a set of weights $W^{\prime}$ such that $\Delta$ is a weighted diagram with site set $t(S)$ and weight set $W^{\prime}$. Hence, diagrams representing $\Delta$ constitute a cone $W \operatorname{Vor}(\Delta)$ whose elements are sets of weighted sites defined up to translation such that their diagrams give partition $\Delta$. A $d$-tension on $\Delta$ defines an element of $W \operatorname{Vor}(\Delta)$ uniquely. The preceding theorems imply the following proposition.

Proposition 7.3. $\quad W \operatorname{Vor}(\Delta)$ is isomorphic to the cone of dual decompositions Dual $(\Delta)$ (up to translation) and to the cone of d-tensions $\operatorname{Tension}_{d}(\Delta)$.

Ash and Bolker [1] proved that a plane decomposition by finite convex polyhedra is a weighted diagram if and only if it is a section of a higher-dimensional Voronoi decomposition. As a non-trivial application of this theorem one can mention that any rhombic Penrose tiling can be represented by a weighted diagram, since it is a section of the standard decomposition of $\mathbb{R}^{5}$ by cubes. In fact, the Ash-Bolker theorem is $d$ dimensional, as their proof does not depend on $d$. There is an easy way to establish a correspondence between weighted Voronoi diagrams and sectional Voronoi diagrams. Assume that all weights are negative. (Since all weights are bounded in absolute value, we can always add to all weights an appropriate constant and make them negative.) A weighted site $s$ with coordinates $\left(x^{1}, \ldots, x^{d}\right)$ and weight $w(s)$ corresponds to a not-weighted site in $\mathbb{R}^{d+1}$ with coordinates $x^{1}, \ldots, x^{d}, \sqrt{-w_{s}}$. Conversely, if $h_{s}$ is the distance between $\mathbb{R}^{d}$ and a site $s$ in $\mathbb{R}^{d+1}$ whose (not-weighted) Voronoi domain intersects $\mathbb{R}^{d}$, then the corresponding site on $\mathbb{R}^{d}$ has the same first $d$ coordinates and weight $-\left|h_{s}\right|^{2}$. The following proposition is a direct consequence of the Ash and Bolker theorem and Theorem 5.1.

Proposition 7.4. A decomposition of $\mathbb{R}^{d}$ by finite convex polyhedra has a d-tension if and only if it is the section of a $(d+1)$-dimensional Voronoi diagram.

The following theorem shows that the classes of weighted Voronoi diagrams and Delaunay decompositions coincide (the author has not found this statement anywhere in literature).

Theorem 7.5. A decomposition $\Delta$ of $\mathbb{R}^{d}$ by convex compact polyhedra is a weighted Voronoi diagram if and only if $\Delta$ is a weighted Delaunay decomposition.

Proof. If $\Delta$ is weighted Voronoi, it has the dual Delaunay decomposition $\Delta^{*}$. A $k$-cell of this decomposition is the convex hall of the weighted Voronoi sites of $d$-cells making full contact in a $(d-k)$-face of $\Delta . S k^{1}(\Delta)$ is a reciprocal for $\Delta^{*}$. We want to show that the 
vertices of $S k^{1}(\Delta)$ can be taken for the sites of a weighted Voronoi diagram representing $\Delta^{*}$. Fix zero weight for a vertex of $S k^{1}(\Delta)$ and then define weights on the other vertices of $S k^{1}(\Delta)$ by weight transfer via edge-paths on $S k^{1}(\Delta)$. Let $v$ and $v_{1}$ be adjacent vertices of $S k^{1}(\Delta)$. If a vertex $v$ is assigned weight $w(v)$ and $x$ is the intersection point of the line spanned by $\left[v v_{1}\right]$ with the plane spanned by the corresponding facet $F$ of $\Delta^{*}$, then the site $v_{1}$ is assigned weight

$$
w\left(v_{1}\right)=\mathbf{d}^{2}\left(x, v_{1}\right)-\mathbf{d}^{2}(x, v)+w(v)
$$

This assignment of weights makes the plane spanned by $F$ equidistant (in terms of weighted distances) from the weighted sites $(v, w(v))$ and $\left(v_{1}, w\left(v_{1}\right)\right)$. A simple check shows that such transfer of weights is well defined over the cycles of $S k^{1}(\Delta)$ corresponding to the stars of all $(d-2)$-cells of $\Delta^{*}$. Therefore by Lemma 4.2 weights can be assigned to all vertices of $S k^{1}(\Delta)$ turning $\Delta^{*}$ into a weighted Voronoi diagram. Sites of a weighted Voronoi diagram are vertices for the dual weighted Delaunay diagram. Therefore vertices of $\Delta$ can be associated with weights so that $\Delta$ can be regarded as a weighted Delaunay decomposition.

Let $\Delta$ be weighted Delaunay. It has the dual weighted Voronoi decomposition $\Delta^{*}$. By Theorems 7.1 and 7.2 $\Delta$ is weighted Voronoi.

The straightforward adaptation of the Euclidean theory of weighted diagrams, duality and tensions given in this section for the spherical case gives a somewhat more symmetric form of the theory for spherical complexes (see also Section 12 and [13] for the twodimensional case).

Theorem 7.6. The following properties of a spherical d-complex $\Delta$ with convex cells are equivalent:

1. $\Delta$ is a weighted Voronoi diagram in $\mathbb{S}^{d}$;

2. $\Delta$ is a weighted Delaunay decomposition of $\mathbb{S}^{d}$;

3. $\Delta$ is the central projection of a convex $(d+1)$-polytope in $\mathbb{R}^{d+1}$;

4. $\Delta$ has a "spherical" d-tension.

\section{Combinatorics of $\mathcal{M}^{d}$ and $\operatorname{Lift}\left(M^{d}\right)$}

The following obvious observations give important implications for the analysis of stresses and liftings of $(d-k)$-primitive manifolds.

Proposition 8.1. Assume that the star St $\left(C^{d-k}\right)$ of a $(d-k)$-cell $C^{d-k}$ in a d-manifold contains $k+1(d-1)$-cells. If $\operatorname{St}\left(C^{d-k}\right)$ is realized in $\mathbb{R}^{d}$ generically (no pair of $(d-1)$ cells lie on the same hyperplane) and the coefficient of $d$-stress for one of its $(d-1)$-cells is fixed, then the values of $d$-stresses for the other $(d-1)$-cells are uniquely determined. If the d-cells of $S t\left(C^{d-k}\right)$ are convex and $S t\left(C^{d-k}\right)$ is embedded, then all coefficients of stresses have the same sign. 
Proposition 8.2. Assume the star $\operatorname{St}\left(C^{d-k}\right)$ of a $(d-k)$-cell $C^{d-k}$ contains $k+1$ $(d-1)$-cells. If $S t\left(C^{d-k}\right)$ is realized in $\mathbb{R}^{d}$ generically and a lifting of the star of a $(d-1)$-cell of $S t\left(C^{d-k}\right)$ is fixed, then the lifting of $\operatorname{St}\left(C^{d-k}\right)$ is uniquely determined. If the d-cells of the star are convex and the star is embedded, then all dihedral angles of the lifting are either convex or concave.

The star of a $(d-2)$-cell can be either embedded or realized with a self-intersection. If the star is $(d-2)$-primitive, then there is only one type of PL-realization with a selfintersection (under our definition of a PL-realization), namely, where two $d$-cells have the same orientation, and the third cell has the opposite one. In the latter case we say that the star is folded.

Let $[x, y]$ be a segment in $\mathbb{R}^{d}$. A combinatorial path $\left[C_{1}, \ldots, C_{n}\right]$ of $d$-cells of a PL-realization $M^{d}$ is strung on $[x, y]$ if:

1. $x \in C_{1}, y \in C_{n}$;

2. $[x, y] \subset \bigcup_{i=1}^{n}\left|C_{i}\right|$;

3. for any $i \neq j,\left|C_{i}\right| \cap\left|C_{j}\right|$ is either a common facet of $C_{i}$ and $C_{j}$ or empty.

Let $L(\mathbf{x} ; C)$ be a lifting of $M^{d}$. If for any $x, y \in\left|M^{d}\right|$ a combinatorial path strung on $[x, y]$ is lifted convex up (resp. down), then $L(\mathbf{x} ; C)$ is called locally convex (resp. concave).

Theorem 8.3. Let $\mathcal{M}^{d}$ be $(d-2)$-primitive, and each $d$-cell of $\mathcal{M}^{d}$ has an internal $(d-2)$-face. Assume that for each d-cell $C$ of $\mathcal{M}^{d}$ set $\left(\partial C \backslash \partial \mathcal{M}^{d}\right)$ is a strongly connected $(d-1)$-pseudomanifold. Let $M^{d}$ be a PL-realization of $\mathcal{M}^{d}$ where all cells are convex. If there is a sharp lifting of $M^{d}$, then for any sub-complex of $M^{d}$ which is actually embedded the lifting is either locally concave or convex. The lifting is unique up to the choice of a d-face and a dihedral angle.

Proof. Let $\left[C_{0}, C_{1}, C_{2}\right]$ be a path of $d$-cells on $\mathcal{M}^{d}$ such that, in the realization $M^{d}$, $\bigcup_{i=0}^{2} C_{i}$ is embedded into $\mathbb{R}^{d}$. Let $L(\mathbf{x} ; C)$ be a sharp lifting. Assume $L(\mathbf{x} ; C)$ is convex on $\left(\left[C_{0}, C_{1}\right]\right)$. It is enough to show that the convexity of $l\left(\left[C_{0}, C_{1}\right]\right)$ implies the convexity of $l\left(\left[C_{1}, C_{2}\right]\right)$. Denote by $E_{1}$ a $(d-2)$-cell shared by $C_{0}$ and $C_{1}$. Consider a $d$-cell sharing $E_{1}$ with $C_{0}$ and $C_{1}$. Let $C_{1}$ and this $d$-cell share a facet $F_{1}$ and let $F_{2}$ be the facet which is common for $C_{1}$ and $C_{2}$. Connect $F_{1}$ and $F_{2}$ by a combinatorial path $\mathfrak{p}$ on $\partial C_{1}$ which consists of internal facets of $\mathcal{M}^{d}$ sharing internal $(d-2)$-cells (such a path exists by the conditions of the theorem). Let $E_{2}$ be a $(d-2)$-cell shared by $F_{2}$ and the preceding $(d-1)$-cell in $\mathfrak{p}$. Since $\mathcal{M}^{d}$ is $(d-2)$-primitive, the type of the realization of a star cannot switch on the surface of $C_{1}$. Therefore $\operatorname{St}\left(E_{2}\right)$ has the same type of realization as $\operatorname{St}\left(E_{1}\right)$. If $\operatorname{St}\left(E_{1}\right)$ is embedded in $\mathbb{R}^{d}$, then the stars of all $(d-2)$-cells from $\mathfrak{p}$ are embedded in $\mathbb{R}^{d}$. Therefore, $\operatorname{St}\left(E_{2}\right)$ is also embedded and both $\operatorname{St}\left(F_{1}\right)$ and $\operatorname{St}\left(F_{2}\right)$ are lifted in the same way. If $S t\left(E_{1}\right)$ is folded, then the stars of all $(d-2)$-cells from $\mathfrak{p}$ are folded. An application of Proposition 8.2 shows that if $S t\left(F_{1}\right)$ is lifted onto a convex dihedral angle, then $\operatorname{St}\left(F_{2}\right)$ is lifted onto a convex dihedral angle too. In both cases $L(\mathbf{x} ; C)$ has necessarily the same type on $\left[C_{1}, C_{2}\right]$ as on $\left[C_{0}, C_{1}\right]$. Therefore, $L(\mathbf{x} ; C)$ is convex on $\left[C_{0}, C_{1}, C_{2}\right]$. 
Corollary 8.4 [36]. If a $(d-2)$-primitive decomposition of $\mathbb{R}^{d}$ by convex polyhedra has a non-trivial lifting, then this lifting is sharp and globally convex or concave. The lifting is unique up to the choice of a supporting plane and a dihedral angle.

As an implication of the above theorem we have the following.

Corollary 8.5. Let $M^{d}$ be a PL-realization in $\mathbb{R}^{d}$ of a $(d-2)$-primitive manifold and let $L^{d}=L(\mathbf{x} ; C)$ be its sharp lifting to $\mathbb{R}^{d+1}$. If

1. $\left|M^{d}\right|$ is convex,

2. for any $\mathbf{x}$ from $\left|M^{d}\right|$ there are exactly two points in $L^{d}$ which project onto $\mathbf{x}$,

3. all cells of $M^{d}$ are convex,

then $L^{d}$ is a convex sphere in $\mathbb{R}^{d+1}$.

An analog of theorem 8.3 for stresses can be formulated as follows.

Proposition 8.6. Let $\mathcal{M}^{d}$ be $(d-2)$-primitive, and each $d$-cell of $\mathcal{M}^{d}$ has an internal $(d-2)$-face. Assume for each $d$-cell $C$ of $\mathcal{M}^{d}$ the set $\partial C \backslash \partial \mathcal{M}^{d}$ is a strongly connected $(d-1)$-pseudomanifold. If the stars of all internal $(d-2)$-cells of $M^{d}$ are generic, then $\operatorname{dim}_{\text {Stress }}\left(M^{d}\right)$ is equal to either 1 or 0.

Notice that even though Stress $_{d}\left(M^{d}\right)$ and $\operatorname{Lift}\left(M^{d}\right)$ are isomorphic to either $\mathbb{R}$ or 0 for any realization of a closed $(d-2)$-primitive manifold $\mathcal{M}^{d}$ with generic stars of $(d-2)$-cells, they need not coincide.

\section{Sharp Liftings of $(d-k)$-Primitive Manifolds}

It is natural to ask when there is a sharp lifting of a "flat" PL-realization $M^{d}$, especially when $M^{d}$ is a decomposition of $\mathbb{R}^{d}$ or a PL-realization of a sphere in $\mathbb{R}^{d}$. In this section we give an improvement on a well-known theorem of Davis [15] on the existence and uniqueness of a sharp lifting for a 0-primitive (simple) cell-decomposition of $\mathbb{R}^{d}, d>2$. We also improve a theorem by Whiteley [48] on the existence and uniqueness of a lifting for PL-realizations of 0-primitive (simple) $d$-spheres $(d>2)$ in $\mathbb{R}^{d}$. If $H_{1}\left(\mathcal{M}^{d}, \mathbb{Z}_{2}\right)=0$, then the existence of a sharp lifting is equivalent to the existence of an all-non-zero stress (see Section 5). As before, we assume that the stars of all $(d-2)$-cells are generic. We also impose a quite natural restriction on the combinatorics of $\mathcal{M}^{d}$.

Let $C$ be a $d$-cell in a $d$-manifold $\mathcal{M}^{d}$ with boundary. We understand by $\operatorname{recl}\left(\partial C \backslash\left(\partial \mathcal{M}^{d} \backslash \partial S k_{d-3}\left(\mathcal{M}^{d}\right)\right)\right)$ a cell-complex which is obtained from $\partial C$ by removing open $(d-1)$ and $(d-2)$-cells which belong to $\partial \mathcal{M}^{d}$, and then augmenting all remaining $(d-1)$-cells of $C$ by their relative boundaries. Informally it means that after removing boundary (with respect to $\left.\mathcal{M}^{d}\right)(d-1)$-cells from $\partial C$ we tear along those $(d-2)$-cells which occur on the boundary of $\mathcal{M}^{d}$ (e.g. see Fig. 4; in this example the manifold is a 3 -cube decomposed into five simplexes and $C=(a b c d))$.

Condition 9.1. For any $d$-cell $C$ the complex $\operatorname{recl}\left(\partial C \backslash\left(\partial \mathcal{M}^{d} \backslash \partial S k_{d-3}\left(\mathcal{M}^{d}\right)\right)\right)$ can be 


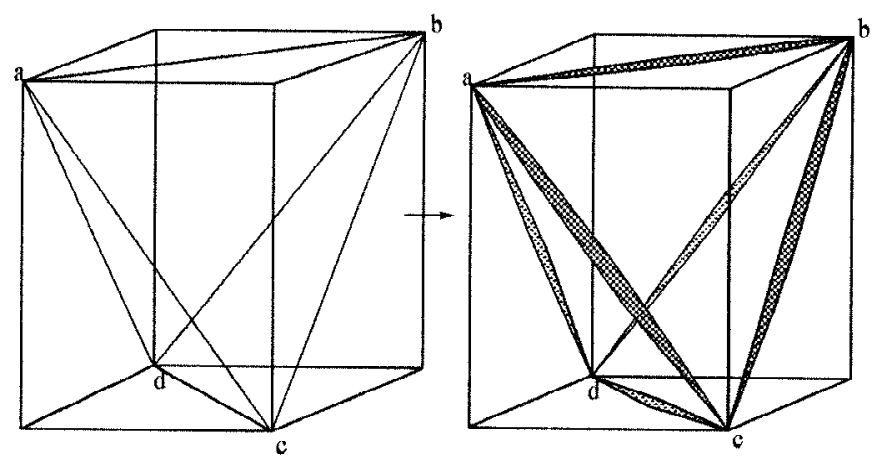

Fig. 4

represented as $\bigcup F_{i}(C)$, where

1. each $F_{i}(C)$ is a $(d-1)$-manifold (possibly with boundary) with $H_{1}\left(F_{i}(C), \mathbb{Z}_{2}\right)=$ 0

2. $F_{i}(C)$ and $F_{j}(C)$ do not share internal $(d-2)$-cells of $\mathcal{M}^{d}$, if $i \neq j$.

Notice that some important classes of manifolds such as cell-partitions of $\mathbb{R}^{d}$, closed compact manifolds, and convex tilings of convex regions in $\mathbb{R}^{d}$ satisfy the above condition. If $d>2$, the local geometric properties of $M^{d}$ (i.e. geometry of the stars) have a stronger effect on $\operatorname{Stress}_{d}\left(M^{d}\right)$ and $\operatorname{Lift}\left(M^{d}\right)$ than in the planar case, as illustrated by the following theorem.

Theorem 9.2. A PL-realization of a $(d-2)$-primitive manifold $\mathcal{M}^{d}(d>2)$ with $H_{1}\left(\mathcal{M}^{d}, \mathbb{Z}_{2}\right)=0$, satisfying Condition 9.1, admits an all-non-zero $d$-stress if and only if the star of each internal $(d-3)$-face has an all-non-zero $d$-stress. If the realization is an embedding and an all-non-zero stress exists, then there is a global tension.

Theorem 9.3. A PL-realization of a $(d-3)$-primitive manifold $\mathcal{M}^{d}(d>2)$ with $H_{1}\left(\mathcal{M}^{d}, \mathbb{Z}_{2}\right)=0$ and satisfying Condition 9.1 has an all-non-zero $d$-stress.

It is easy to see that Theorem 9.3 easily follows from Theorem 9.2, since a generic realization of the star of a $(d-3)$-cell with only four $d$-cells always has an all-non-zero stress which is unique up to scale.

Proof of Theorem 9.2. We call a subset of facets of $\mathcal{M}^{d}$ an independent component if stresses on the facets of this subset do not depend on the choice of stresses for the facets which do not belong to this subset. All internal facets can be partitioned into independent components $I_{k}$. Choose a facet in each component and fix an arbitrary non-zero stress for each chosen facet. We have to show that the fixed set of stresses uniquely determines an all-non-zero stress on all of $M^{d}$. To prove this we translate stress via chains of adjacent 
facets. The stress transfer has to be defined independent of path. By Proposition $8.2 \mathrm{a}$ stress on a facet belonging to the primitive star of a $(d-2)$-cell determines the coefficients of stresses of the other two facets. Therefore, if $F$ and $F^{\prime}$ are two adjacent facets, then there are two non-trivial reciprocal linear maps $l\left(\left[F, F^{\prime}\right]\right)$ and $l\left(\left[F^{\prime}, F\right]\right)$ associated with pairs $\left[F, F^{\prime}\right]$ and $\left[F^{\prime}, F\right]$. Consider a graph $G$ whose vertices are internal facets of $\mathcal{M}^{d}$, and whose edges are internal $(d-2)$-cells of $\mathcal{M}^{d}$, where an edge exists between facet $F$ and $F^{\prime}$ precisely when $F$ and $F^{\prime}$ share a common internal $(d-2)$-cell. (Notice that $G$ is not the dual graph $\mathcal{G}\left(\mathcal{M}^{d}\right)$.) The process of assigning coefficients of stresses to facets of $\mathcal{M}^{d}$ can be regarded as the process of assigning real numbers to vertices of $G$ via the use of functions $l\left(\left[F, F^{\prime}\right]\right)$. In this model, each edge of $G$ is associated with a pair of reciprocal linear functions. $G$ consists of connected components $G_{k}$ corresponding to $I_{k}$ (see above). For each $G_{k}$, we assign a real number $s_{k} \in \mathbb{R}$ to an arbitrarily chosen vertex of $G_{k}$. We have to show that real numbers can be assigned to all vertices of $G_{k}$ so that the numbers corresponding to adjacent vertices are connected by the pair of linear maps assigned to their common edge. This is the same as showing that quality transfer over each cycle is well defined. We can regard $G$ as a sub-graph of the 1-skeleton of the dual (combinatorial) cell-decomposition of $\mathcal{M}^{d}$. Denote by $\mathcal{D}$ this dual decomposition. Since $\mathcal{M}^{d}$ is $(d-2)$-primitive, all 2-cells of $S k^{2}(\mathcal{D})$ corresponding to internal $(d-2)$-cells are (combinatorially) triangles. Such 2-cells of $S k^{2}(\mathcal{D})$ can be decomposed into four smaller triangles, one of them being a cycle of $G$. Let $(A B C)$ be a triangle of $\mathcal{D}$ where the vertices correspond to $d$-cells $A, B$ and $C$ forming the star of an internal $(d-2)$-cell and the edges correspond to the facets of this star. If $e, f$ and $g$ are the "centers" (in the combinatorial sense) of the sides $A B, B C$ and $C A$, then the sub-triangulation of $(A B C)$ consists of $(e f g),(e A g),(e B f),(f C g)$, where (efg) is a cycle of $G$ (see Fig. 5).

In a similar way we decompose all 2-cells of $S k^{2}(\mathcal{D})$ corresponding to boundary $(d-2)$-cells of $\mathcal{M}^{d}$. If the star of a boundary $(d-2)$-cell contains $m d$-cells, then the subdivision of the corresponding 2-cell of $S k^{2}(\mathcal{D})$ consists of $m$ triangles and one $(1+m)$ gon. Consider an arbitrary 1-cycle $\mathfrak{c}$ on $G$. Since $H_{1}\left(S k^{2}(\mathcal{D}), \mathbb{Z}_{\in}\right)=1$, then $\mathfrak{c}=\partial \Delta^{2}$, where $\Delta^{2}$ is a 2-chain from $\mathcal{C}_{2}\left(S k^{2}(\mathcal{D}), \mathbb{Z}_{\in}\right)$. The chain $\Delta^{2}$ can be represented as the sum of 2-cells from the subdivision of $S k^{2}(\mathcal{D})$ described above. Cells of this subdivision of $S k^{2}(\mathcal{D})$ can be conveniently partitioned into two groups, namely, those which are of type

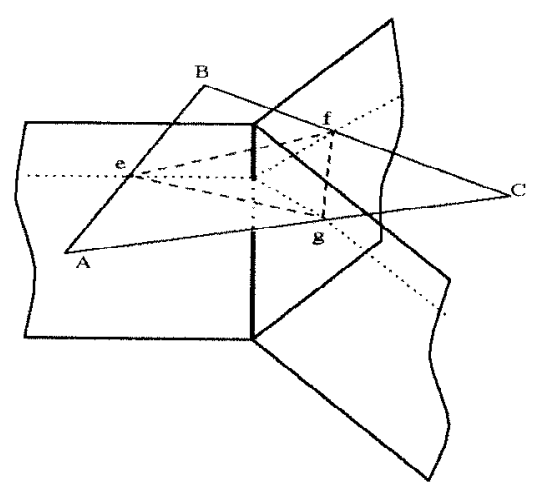

Fig. 5 
(efg) (the boundaries of such cells are the links of internal $(d-2)$-cells of $\left.\mathcal{M}^{d}\right)$ and those which are of type $(e A g)$ (see above). Therefore $\Delta^{2}=\Delta_{1}^{2}+\Delta_{2}^{2}$, where $\Delta_{1}^{2}$ is the sum of 2-cells of the first type and $\Delta_{2}^{2}$ is the sum of 2-cells of the second type. The boundaries of 2-cells of the first type are actually cycles of $G$ corresponding to internal stars of $(d-2)$-cells of $M^{d}$ (clearly, 2-cells that correspond to boundary $(d-2)$-cells of $\mathcal{M}^{d}$ do not occur in $\left.\Delta_{1}^{2}\right)$. We refer to such cycles as link-cycles. Let $\partial \Delta_{2}^{2}=\sum_{k} \mathfrak{c}\left(C_{k}^{d}\right)$, where $\mathfrak{c}\left(C_{k}^{d}\right)$ is a cycle connecting only the facets of the $d$-cell $C_{k}^{d}$. We call such cycles surface cycles, for they can be thought of as lying on the surfaces of $d$-cells. Since the choice of the cycle $c$ was arbitrary, the link-cycles and the surface cycles form a generating system of $H_{1}\left(G, \mathbb{Z}_{2}\right)$. We only need to prove that quality transfer is well defined over surface cycles. One can think of quality transfer over a surface cycle connecting facets of a $d$-cell $C^{d}$ as of quality transfer over paths of $(d-1)$-cells of $\partial C^{d}$. Lemma 4.2 provides sufficient conditions that quality transfer on a manifold with $H_{1}=0$ over $\mathbb{Z}_{2}$ is well defined. Before applying Lemma 4.2 we need to obtain some information on circuits over the links of $(d-3)$-cells on $\partial C^{d}$. By Condition $9.1 \operatorname{recl}\left(\partial C^{d} \backslash\left(\partial \mathcal{M}^{d} \backslash \partial S k^{d-1}\left(\mathcal{M}^{d}\right)\right)\right)=\bigcup F_{m}\left(C^{d}\right)$, where for each manifold $F_{m}\left(C^{d}\right)$ all $(d-3)$-cells internal with respect to $F_{m}\left(C^{d}\right)$ are internal with respect to $\mathcal{M}^{d}$. By hypothesis, there is an all-non-zero stress for the star of each internal $(d-3)$-cell of $\mathcal{M}^{d}$ which is, of course, unique up to scale. It implies that the quality transfer over the link of each internal $(d-3)$-cell of $\partial C^{d} \backslash \partial \mathcal{M}^{d}$ is well defined. Quality transfer is well defined on $\partial C^{d} \backslash \partial \mathcal{M}^{d}$ if and only if it is well defined on each $F_{m}\left(C^{d}\right)$. By Condition 9.1, all such components are manifolds with $H_{1}=0$ over $\mathbb{Z}_{2}$. By Lemma 4.2 , quality transfer over every circuit of $(d-1)$-cells of $F_{m}\left(C^{d}\right)$ is well defined. Therefore, quality transfer is well defined over surface cycles. Thus it is well defined on $G$. It therefore follows that an all-non-zero stress on $M^{d}$ exists and is unique up to scale for each independent component $I_{k}$.

Corollary 9.4. A PL-realization of a $(d-2)$-primitive $\mathcal{M}^{d}(d>2)$ satisfying Condition 9.1 and $H_{1}\left(\mathcal{M}^{d}, \mathbb{Z}_{2}\right)=0$, admits a sharp lifting if and only if the star of each $(d-3)$-face has a sharp lifting. If $\mathcal{M}^{d}$ is closed, then any non-trivial lifting is sharp and unique up to the choice of an affine function and a dihedral angle.

Assume all cells of $M^{d}$ are convex. Under the conditions of the preceding theorem, any sharp lifting is either convex or concave on each sub-complex of $\mathcal{M}^{d}$ which is embedded into $\mathbb{R}^{d}$.

Corollary 9.5. Any PL-realization of a $(d-3)$-primitive $\mathcal{M}^{d}(d>2)$ satisfying Condition 9.1 and $H_{1}\left(\mathcal{M}^{d}, \mathbb{Z}^{2}\right)=0$ has a sharp lifting. If the realization is an embedding and all cells are convex, then there is a convex lifting.

These results improve a well-known theorem of Davis [15] on the existence and uniqueness of a convex lifting for a 0 -primitive (simple) finite decomposition of $\mathbb{R}^{d}$ into convex polyhedra as well as a similar theorem by Whiteley [48] for spheres. Combining results of Theorem 8.3 and the above corollaries we obtain the following result generalizing a theorem by Whiteley [48], where the manifold is required to be 0 -primitive. 
Theorem 9.6. Let $M^{d}(d>2)$ be a PL-realization of a $(d-3)$-primitive manifold. If

1. $\left|M^{d}\right|$ is convex,

2. the realization $M^{d}$ covers each point of the interior of $\left|M^{d}\right|$ twice,

3. all cells of $M^{d}$ are convex,

then $M^{d}$ has a unique lifting (up to the choice of a supporting $d$-plane and a dihedral angle) onto a convex sphere in $\mathbb{R}^{d+1}$.

\section{Algorithmic Analysis of Stresses and Liftings}

It is useful to consider liftings from a somewhat more general point of view, adopted in a field of artificial intelligence called scene analysis of polyhedral pictures (see [39]).

A polyhedral incidence structure is a triple $S=(V, F, I)$ : an abstract set of vertices $V$, an abstract set of faces $F$, and a set of incidences $I \subset V \times F$, where $V \times F$ denotes the set of all ordered pairs whose first elements are taken from $V$ and second elements from $F$.

A polyhedral $d$-picture is a pair $(S, \mathbf{p})$ : an incidence structure $S$ and a location map p: $V \rightarrow \mathbb{R}^{d}$ assigning coordinates to vertices. A lifting is a pair of "lifting" maps $(L F, L P)$ : a map $L F: F \rightarrow \mathbb{R}^{d+1}, f \mapsto A^{f}$ assigning affine functions on $\mathbb{R}^{d}$ to faces, and a map $L P: V \rightarrow \mathbb{R}, \mathbf{p}(v) \mapsto z^{\mathbf{p}(v)}$ assigning points in $\mathbb{R}^{d+1}$ to vertices of $V$, such that, for every $v \in V$ and for every incidence $(v, f), z^{\mathbf{p}(v)}=A^{f}(\mathbf{p}(v))$. Liftings of $(S, \mathbf{p})$ form a linear space $\operatorname{Lift}(S, \mathbf{p})$. A lifting is sharp if any two affine functions corresponding to faces with at least $d$ common vertices are distinct.

It is worth mentioning that the problem of recognizing whether a planar polyhedral picture can be interpreted as the projection of a spatial polyhedral scene satisfying given constraints is one of the central problems in computer line drawing [39]. Similar problems for parallel drawings occur in computer-aided geometric design (see [50]). Since parallel drawings in $\mathbb{R}^{d}$ are equivalent to liftings from $\mathbb{R}^{d}$ to $\mathbb{R}^{d+1}$ (see [50]) the ability to find a lifting of a given type allows us to construct a parallel drawing having prescribed qualitative properties. Thus algorithms constructing liftings may be useful to computeraided design and computer vision [50], [39]. As an example of another application for algorithms of this sort one can mention perturbing a non-simple decomposition by convex polyhedra of a region in $\mathbb{R}^{d}(d>2)$. If we do not know of any sharp lifting for the decomposition, then we may not be able to perturb the decomposition so that it will become 0 -primitive (simple). However, if there is a method to find a sharp lifting, one can always perturb such a lifting and project it back to $\mathbb{R}^{d}$. The perturbed decomposition will be 0 -primitive and the convexity of the cells will be preserved. By the results of Sections 7 and 9 a 0 -primitive decomposition of $\mathbb{R}^{d}(d>2)$ is always a weighted Voronoi diagram; thus algorithms constructing sharp liftings can be used to approximate decompositions admitting sharp lifting by weighted Voronoi diagrams (see [40] and [41] for applications of such approximations). Since Tension ${ }_{d}\left(M^{d}\right) \cong W \operatorname{Vor}\left(M^{d}\right)$ for cell-decompositions of $\mathbb{R}^{d}$ by convex polyhedra, the problem of finding an all-non-zero stress is equivalent to the problem of recognition of weighted diagrams and sections of Voronoi diagrams. It can be useful in mathematical crystallography and other sciences dealing with space partitions [40], [41]. 
A picture $(S, \mathbf{p})$ is called generic if the dimension of the space of liftings of $(S, \mathbf{p})$ is minimal over all realizations $\mathbf{p}$ of the incidence structure $S$ in $\mathbb{R}^{d}$. All other realizations are called singular. Generic realizations form an open dense subset of the space $\mathbb{R}^{|d V|}$ of all $\mathbb{R}^{d}$-realizations of $S$. Singular realizations correspond to points of an algebraic variety in the space of all $\mathbb{R}^{d}$-realizations. This variety is called the algebraic variety of singular realizations of $S$ (for details see [49]).

There is a polynomial time combinatorial (i.e. not employing realization data) algorithm by Sugihara which determines whether a generic picture has a sharp lifting, and computes the dimension of the space of lifting in this case (see [25], [39], [46], and [47]). In fact, one can modify this algorithm so that it will find $\operatorname{dim} \operatorname{Lift}(S, \mathbf{p})$ for any generic picture $(S, \mathbf{p})$. Imai's modification [25] of this algorithm has complexity $O\left(|I|^{2}\right)$. It is an open question whether there is a polynomial algorithm which computes all maximal subpictures of $(S, \mathbf{p})$ which have sharp liftings. Note that polyhedral pictures which occur in practical applications usually have symmetries or traces of symmetries which often make the use of combinatorial methods inappropriate for practical implementations.

In this paper we consider a special case, where $S$ is the $d$-cell-vertex incidence structure of a fixed cell-decomposition of a manifold $\mathcal{M}^{d}$. Since we want every $k$-cell to be realized as a PL-ball living in an affine $k$-subspace of $\mathbb{R}^{d}$, it is natural to mean by the space of $\mathbb{R}^{d}$-realizations of $\mathcal{M}^{d}$ an affine subspace of $\mathbb{R}^{d f_{0}}$ which consists of all realizations satisfying this requirement. If $\mathcal{M}^{d}$ is simplicial, then this space is all $\mathbb{R}^{d f_{0}}$. Indeed, from a practical point of view the cases of orientable 2-manifolds and 3-manifolds are the most important ones (see [39], [50], and [49]). We prescribe the type of dihedral angle of a lifting for some edges of a picture (e.g. convex versus concave, if two adjacent cells lie in different half-planes) and ask whether there is a lifting which satisfies these conditions. More formally, the lifting problem is as follows:

1. Find $\operatorname{dim} \operatorname{Lift}\left(M^{d}\right)$.

2. Given a set of restrictions for some dihedral angles, determine whether a lifting of this type exists and construct such a lifting if it does.

For different generic realizations of a simplicial $\mathcal{M}^{d}$ the answers to the second part of the lifting problem may be different, and therefore there are no purely combinatorial methods to answer the above questions for general instances of this problem [10]. This is because the set of generic $\mathbb{R}^{d}$-realizations of $\mathcal{M}^{d}$ admitting the same type of lifting is a semi-algebraic subvariety of full dimension in the space of all $\mathbb{R}^{d}$-realizations of $\mathcal{M}^{d}$. Notice that similar questions arise in the theory of tensegrity frameworks [11], [50].

One can express qualitative restrictions on dihedral angles in terms of $C_{1}^{0}$-cofactors (see Section 5). Let $A$ and $B$ be $d$-cells of $M^{d}$ which make contact in a facet $F$. Notice that $c_{1}^{0}(A, B)>0$ if and only if the affine function $L(A, \mathbf{x})$ corresponding to $A$ is greater that the affine function $L(B, \mathbf{x})$ corresponding to $B$ on the half-space $\langle\mathbf{x}, \mathbf{n}\rangle+c<0$, where $\langle\mathbf{x}, \mathbf{n}\rangle+c=0$ is the supporting hyperplane of $F$ and $n$ is the outer normal to $A$ at $F$. For example, we consider the case when $d$-cells $A$ and $B$ are convex (in fact, this is not important for our definitions). When $A$ and $B$ lie in different half-spaces with respect to the supporting hyperplane of $F, c_{1}^{0}(A, B)>0$ if and only if the lifting of $\operatorname{St}(F)$ is convex, and $c_{1}^{0}(A, B)<0$ if and only if the lifting of $\operatorname{St}(F)$ is concave. When $A$ and $B$ lie in the same half-space, then $c_{1}^{0}(A, B)>0$ if and only if the cell of the lifting corresponding to $A$ lies above the cell of the lifting corresponding to $B$. The above restrictions (for 


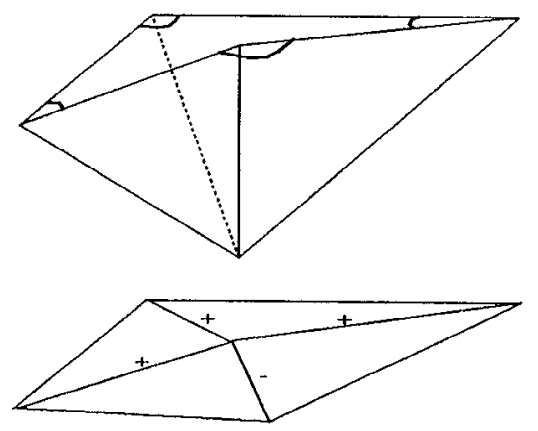

Fig. 6

$d=2$ ) are standard in computer vision (see [39]), and, when $\operatorname{St}(F)$ is embedded, are usually expressed with edge labels + for convex lifting and - for concave lifting (e.g. see Fig. 6). It is easy to see now that liftings which satisfy some fixed restrictions of the above type form an open convex polyhedral cone in the linear space of liftings.

If $\mathcal{M}^{d}$ is oriented, then restrictions of the above type naturally give rise to dependent sets of an oriented matroid on internal facets of $\mathcal{M}^{d}$. Fix an orientation and a lifting for $M^{d}$. For a facet $F$ shared by $d$-cells $A$ and $B$ define the sign of $F$ in the matroid of liftings by $o(A) \operatorname{sgn}\left(c_{1}^{0}(A, B)\right)$. Facets with non-zero signs form a dependent set of the matroid. Hence, in the case of orientable $M^{d}$ it can be said that the purpose of the algorithm is to determine whether a dependent set with given signing exists.

Let $\mathcal{I}\left(\mathcal{M}^{d}\right)$ be the incidence graph of a finite cell-decomposition of a manifold $\mathcal{M}^{d}$ (see [20] for a description of incidence graphs), and let, as before, $M^{d}$ be a PL-realization of $\mathcal{M}^{d}$ in $\mathbb{R}^{d}$. To store the parameters of the realization $M^{d}$ with the incidence graph of combinatorial $\mathcal{M}^{d}, \mathcal{I}\left(\mathcal{M}^{d}\right)$ should be equipped with additional information which determines the positions of facets and $d$-cells of $M^{d}$ (up to translation). Assume that in the incidence graph $\mathcal{I}\left(M^{d}\right)$ storing the PL-realization $M^{d}$, each node representing a facet of $M^{d}$ stores the coefficients of a normal to the hyperplane on which the PLrealization of this facet lies. Since non-simplicial, non-convex and non-compact cells are allowed, this information is not sufficient for efficient algorithms. If $C$ is a $d$-cell and $F$ is its facet, the data structure should allow determining, in constant time, from which side of the facet $F$ the cell $C$ makes contacts with $F$ (when $C$ is a simplex, it can always be determined in constant time). We modify $\mathcal{I}\left(M^{d}\right)$ by restructuring the layer of the nodes representing the facets. For a node representing a facet with normal $\left(a_{1}, \ldots, a_{d}\right)$ we create a node-antipode representing the same facet, but with normal $\left(-a_{1}, \ldots,-a_{d}\right)$. Let $n\left(F ;\left(a_{1}, \ldots, a_{d}\right)\right)$ be a node of $\mathcal{I}\left(M^{d}\right)$ representing a facet $F$ with equation $a_{1} x^{1}+\cdots+a_{d} x^{d}+c=0$, and let $F$ be shared b y $d$-cells $C_{1}$ and $C_{2}$. The node $n\left(F ;\left(a_{1}, \ldots, a_{d}\right)\right)$ and a node that represents $C_{i}$ are linked by an arc in the modified graph if and only if $\vec{a}=\left(a_{1}, \ldots, a_{d}\right)$ is an outer normal to $C_{i}(i=1,2)$. In other words, in the modified incidence graph $\mathcal{I}\left(\mathcal{M}^{d}\right)$ (we do not change the notation) the layer which represents the facets contains two copies of each facet with different orientations 
of normals. In fact, the first algorithm uses only a subgraph of $\mathcal{I}\left(M^{d}\right)$ which stores the nodes representing $d$-cells, $(d-1)$-cells and all incidences between them. Denote this subgraph by $\mathcal{I}_{d-1}\left(M^{d}\right)$. The second algorithm uses $\mathcal{I}_{d-2}\left(M^{d}\right)$ which contains the nodes representing $d$-cells, $(d-1)$-cells, $(d-2)$-cells and all incidences between them. In the description of the algorithms we also use the combinatorial dual graph $\mathcal{G}=(\mathcal{V}, \mathcal{E})$ of $\mathcal{M}^{d}$ introduced in Section 6. Whenever it is appropriate, we identify in our descriptions $d$-cells of $\mathcal{M}^{d}$ with vertices of $\mathcal{G}$, and facets of $\mathcal{M}^{d}$ with edges of $\mathcal{G}$.

\section{Algorithm for $(d-2)$-Primitive Decompositions}

\subsection{Preliminaries to Algorithm 1}

A reciprocal is called maximum if it has the maximum possible number of non-degenerate edges. The algorithm attempts to construct a maximum reciprocal for $M^{d}$ and finds the dimension of $\operatorname{Rec}\left(M^{d}\right)$ in linear running time in the number of internal facets (recall that by the results of Section $\left.6 \operatorname{Rec}\left(M^{d}\right) \equiv \operatorname{Lift}\left(M^{d}\right)\right)$.

Designing Algorithm 1 we were guided by the following analog of Theorem 8.3 for reciprocals. This proposition is a direct consequence of the Theorems 6.1 and 9.3.

Proposition 11.1. Let $\mathcal{M}^{d}$ be $(d-2)$-primitive, and let each $d$-cell of $\mathcal{M}^{d}$ have an internal $(d-2)$-face. Assume for each $d$-cell $C$ of $\mathcal{M}^{d}$ that the set recl $\left(\partial C \backslash \partial \mathcal{M}^{d}\right)$ is a strongly connected $(d-1)$-pseudomanifold. If the stars of all internal $(d-2)$-cells of $M^{d}$ are generic, then $\operatorname{dim} \operatorname{Rec}\left(M^{d}\right)$ is equal to either 1 or 0.

Since the correctness of the algorithm can be guaranteed only for $(d-2)$-primitive decompositions, we assume that our input is a PL-realization of a finite $(d-2)$-primitive cell-decomposition of a manifold. We assume also that realization $M^{d}$ has generic stars of $(d-2)$-cells and that the combinatorial $\mathcal{M}^{d}$ satisfies at least one of the following conditions:

1. $\mathcal{M}^{d}$ is a closed manifold,

2. $H_{1}\left(\mathcal{M}^{d}, \mathbb{Z}_{2}\right)=0$.

In fact one can adopt the algorithm so that it handles non-generic stars of $(d-2)$-cells, but we omit the proof. We pay attention to $d$-cells with parallel facets since such cells occur in tilings derived from lattices and other point systems with symmetries, and it seems quite natural to take them into account. The applicability of the algorithm to the above classes, its complexity and its robustness are discussed in Sections 11.3 and 11.4.

The above proposition implies that the space of liftings is determined by a system of at most $f_{d-1}^{\circ}\left(\mathcal{M}^{d}\right)$ linear equations like $x_{i}=c x_{j}$, where $x_{i}, x_{j}$ are cofactors of the stars of facets, and $k$ or $-k$ is the length of the corresponding edge of a maximal reciprocal. It is clear, however, that one can read off a system of equations determining $\operatorname{Lift}\left(M^{d}\right)$ from a maximum reciprocal and $\mathcal{I}_{d-1}\left(M^{d}\right)$ (see Section 10) in time $O\left(f_{d-1}^{\circ}\left(\mathcal{M}^{d}\right)\right)$. 
In this coordinate system, the cone of liftings of a certain fixed type is determined by inequalities like $x_{i}<0$ or $x_{i}<0$, and therefore the question of the existence of a lifting of a certain type can be answered in time $O\left(f_{d-1}^{\circ}\left(\mathcal{M}^{d}\right)\right)$. Thus our algorithm solves the lifting problem (see Section 10) for $(d-2)$-primitive decompositions of closed manifolds and homology disks in optimal time.

As in the previous section, we call a subset of internal facets of $\mathcal{M}^{d}$ an independent component if stresses on the facets of this subset do not depend on the choice of stresses for the facets from the complement of this subset. For example, if two facets in a $(d-2)$ primitive manifold can be connected by a chain of adjacent facets such that every two consecutive facets share an internal $(d-2)$-cell, then they belong to the same component. Often it is somewhat more convenient to describe the dependences of stresses via the use of the combinatorial dual graph $\mathcal{G}$. We switch freely between vertices and edges of $\mathcal{G}$ and $d$-cells and facets of $\mathcal{M}^{d}$. Call a subgraph $\mathcal{G}^{\prime}=\left(\mathcal{V}^{\prime}, \mathcal{E}^{\prime}\right)$ of $\mathcal{G}=(\mathcal{V}, \mathcal{E})$ an independent component if the choice of stresses for facets from $\mathcal{E}^{\prime}$ does not depend on the choice of stresses for facets from $\mathcal{E}-\mathcal{E}^{\prime}$. All facets can be partitioned into disjoint independent components $I_{k}$ in a unique way. This partition corresponds to an edgepartition of $\mathcal{G}$.

Starting with an arbitrary edge of $\mathcal{G}$, the algorithm processes an independent component of $\mathcal{G}$ to which this edge belongs. In the same way the algorithm processes all other independent components of $\mathcal{G}$ in a sequence. As the algorithm works, a dynamical data structure LIST which contains all not-processed $d$-cells of $\mathcal{M}^{d}$ (i.e. their indexes) is maintained. Initially LIST contains $d$-cells of $\mathcal{M}^{d}$. We maintain links (implemented as pointers in both directions) between facets of $\mathcal{M}^{d}$ and corresponding elements of LIST. Thus, we can assume that any $d$-cell which is known to be in LIST can be removed from LIST in constant time, and that for any $d$-cell of $\mathcal{M}^{d}$ one can check in constant time whether its index is in LIST. (When an element of LIST is deleted, its link is deleted too.) The algorithm uses STACK where $d$-cells are put as the algorithm proceeds. Initially, STACK is empty. The algorithm attempts to construct a reciprocal for each independent component of $\mathcal{G}$ using an inductive procedure which finds vertices of the reciprocal. Let $\mathcal{G}^{\prime}$ be an independent component of $\mathcal{G}$, and let $R_{k}^{\prime} \subset R^{\prime} \subset R(k>2)$ be the subgraph of $R$ corresponding to the first $k$ constructed vertices of $\mathcal{G}^{\prime}$. Let $v(A) \in \mathbb{R}^{d}$ denote the vertex of $R_{k+1}^{\prime}$ corresponding to a $d$-cell $A$. Assume that vertices $v(B)$ and $v(C)$ of $R_{k}^{\prime}$ corresponding to $d$-cells $B$ and $C$ that are adjacent to $A$ have been constructed on the first $k$ steps, and the construction can be extended to $A$. In this case one can find vertex $v(A)$ as the intersection point of the lines passing through $v(B)$ and $v(C)$, and perpendicular to the facets at which $B$ and $C$ contact $A$. Denote by $V(A ; v(B), v(C))$ the function expressing this dependence (we write $V(A ; v(B), v(C))=\emptyset$ if these lines do not intersect). If $v(A)$ does not exist, $R_{k+1}^{\prime}$ and therefore reciprocal $R^{\prime}$ corresponding to $\mathcal{G}^{\prime}$ collapses into a single point. After computing function $V(A ; v(B), v(C)) d$-cell $A$ is marked as processed (in both cases). If $R^{\prime}$ collapses into a point, all $d$-cells corresponding to its vertices are marked as processed. The algorithm produces the coordinates of vertices of a maximum reciprocal $R$ and computes $\operatorname{dim} \operatorname{Rec}\left(M^{d}\right)$. It also formulates a system of linear equations $\Theta$ determining $\operatorname{Lift}\left(M^{d}\right)$ whose variables are the $C_{1}^{0}$-cofactors (in our notation variable $x_{A B}$ represents cofactor $c_{1}^{0}(A, B)$ ). Let $a(C)$ be the number of actually processed $d$-cells which are adjacent to $C$ (this number changes as the algorithm proceeds). Initially, for all $C$ the $a(C)$ are set equal to 0 . Index $j$ ranges over all 
independent components and is initialized by 1 . The dimension of the $j$ th component is denoted by $\operatorname{dim}_{j}$. Both $\operatorname{dim} \operatorname{Rec}\left(M^{d}\right)$ and $\operatorname{dim}_{j}$ are initialized to zero.

\subsection{Algorithm 1}

0 while $L I S T$ is not empty;

1 if $S T A C K$ is not empty then take a cell $A$ from $S T A C K$; mark $A$ as processed; remove $A$ from $L I S T$;

2 else if $L I S T$ is empty; then $\operatorname{dim} \operatorname{Rec}\left(M^{d}\right):=\operatorname{dim} \operatorname{Rec}\left(M^{d}\right)+\operatorname{dim}{ }_{j}$; terminate else remove a cell $A$ from $L I S T$;

if there is a cell $B$ adjacent to $A$ in the LIST then remove $B$ from LIST; push $A$ onto $S T A C K$; set $v(B)$ at the origin; mark $B$ as processed;

else mark $A$ as processed; $\operatorname{dim} \operatorname{Rec}\left(M^{d}\right):=\operatorname{dim} \operatorname{Rec}\left(M^{d}\right)+1$; $\mathrm{j}:=\mathrm{j}+1 ; \operatorname{dim}_{j}:=1$; denote $x_{A B}$ by $x_{j}$

\section{endif}

\section{endif} endif

3 if $a(A) \geq 2$ then scan all $d$-cells adjacent to $A$ and find two processed cells $D$ and $E$ that make contact with $A$ in non-parallel facets;

if $V(A ; v(D), v(E))$ exists then add " $x_{A D}=\langle v(A) v(D), n(A, D)\rangle x_{j}$ " and " $x_{A E}=\langle v(A) v(E), n(A, E)\rangle x_{j}$ " to $\Theta$; else $\operatorname{dim}_{j}:=0$; add " $x_{j}=0$ " to $\Theta$;

for each $C$ which is a processed neighbour of $A$ add " $x_{A C}=0$ " to $\Theta$;

\section{endif}

\section{endfor}

else if $a(A)=1$ then find the processed $d$-cell adjacent to $A$ (denote it by $F$ ); find the common facet $C^{d-1}$ of $A$ and $F$; find a point in $\mathbb{R}^{d}$ at unit distance from $v(A)$ so that $[v(A) v(F)]$ is orthogonal to $C^{d-1}$

\section{endif}

\section{endif}

4 for all $d$-cells $G$ adjacent to $A$ do

if $G$ has not been processed then $a(G)=a(G)+1$

if $G$ has two adjacent $d$-cells which make contact with $G$ in two non-parallel facets then push $G$ onto STACK

\section{endif}

elseif $v(A)$ does not exists or $[v(G) v(A)]$ is not orthogonal to the corresponding facet endif then $\operatorname{dim}_{j}:=0$; add " $x_{j}=0$ " to $\Theta$; add " $x_{A G}=0$ " to $\Theta$;

\section{endfor}

5 end 


\subsection{Analysis of Algorithm 1}

The algorithm is supposed to process all independent components in a sequence, and can give wrong results only if it does not recognize independent components properly, i.e. if it treats edges of $\mathcal{G}$ which belong to a component as edges from different components. It is enough to prove that STACK does not become empty until all $d$-cells of a current component $\mathcal{G}_{1}=\left(\mathcal{V}_{1}, \mathcal{E}_{1}\right)$ have been processed. The algorithm recognizes $\mathcal{G}_{1}$ as processed before all $d$-cells corresponding to vertices of $\mathcal{E}_{1}$ have been processed only if cannot find a non-processed cell which has two processed neighbours in non-parallel facets. The construction of the algorithm guarantees that in this case there is a $d$-cell $C$ with one processed neighbour corresponding to a vertex of $\mathcal{G}_{1}$. Let $B$ be a processed neighbour of $C$, and let $A$ be a processed neighbour of $B$ from $\mathcal{G}_{1}$. Note that $B$ must have a processed neighbour, because of the assumptions about $\mathcal{G}_{1}$ and $C$.

First, consider the case when $\mathcal{M}^{d}$ is closed. Then $A$ and $C$ can be connected by a path which consists of $d$-cells making full contact with $B$. Therefore one can find a nonprocessed $d$-cell which contacts two processed $d$-cells (one of them is $A$ ) and shares an internal $(d-2)$-cell with them. It contradicts our assumption that the STACK is empty.

Now assume that $H_{1}\left(\mathcal{M}^{d}, \mathbb{Z}_{2}\right)=0$. Since $C \in \mathcal{V}_{1}$, there is a path $\mathfrak{p}$ in $\mathcal{G}_{1}$ which connects vertices $B$ and $C$, but does not contain edge $[B C]$. We can assume that this path is the sum of two paths $\mathfrak{p}_{1}=\left[B, \ldots, C^{\prime}\right]$ and $\mathfrak{p}_{2}=\left[C^{\prime}, \ldots, C\right]$, where the first consists only of processed vertices except the last vertex, and the second contains only non-processed vertices. Therefore there is a non-trivial edge-cycle in $\mathcal{G}_{1}$ which contains $[B C]$. Since $H_{1}\left(\mathcal{M}^{d}, \mathbb{Z}_{2}\right)=0, \mathfrak{p}_{1}+\mathfrak{p}_{2}+[C B]=\partial \sum_{i} \mathfrak{t}_{i}(\bmod 2)$, where the $\mathfrak{t}_{i}$ are triangles corresponding to the stars of internal $(d-2)$-cells of $M^{d}$ (see Section 4$)$. Let $\sum_{i} \mathfrak{t}_{i}=\sum^{1}+\sum^{2}$, where $\sum^{1}$ is the sum modulo 2 of the $\mathfrak{t}_{i}$ with all processed vertices and $\sum^{2}$ is the sum modulo 2 of the $\mathfrak{t}_{i}$ with at least one non-processed vertex. Assume that none of the triangles of the second type contains two processed vertices. Thus $\partial \sum^{1}=\mathfrak{p}_{1}$, which is impossible since $\mathfrak{p}_{1}$ is not a cycle. Therefore there is a non-processed vertex of $\mathcal{G}_{1}$ which forms a triangle in $\mathcal{G}_{1}$ with two processed vertices. This contradicts our choice of $C$. Since the choice of $\mathcal{G}_{1}$ was arbitrary, one can conclude that the algorithm processes all independent components properly.

It is easy to see that if $L$ is the binary size of the numerical input (an array of the coordinates of normals to facets), then the number of arithmetical operations which have to be performed to a precision of $O(L)$ is $O\left(f_{d-1}^{\circ}\left(\mathcal{M}^{d}\right)\right)$.

\subsection{Robustness and Approximations}

Notice, that even when $\mathcal{M}^{d}(d>2)$ satisfies all of the conditions required for the successful performance of the algorithm (see Section 11.1), a real numerical experiment with not-exact machine arithmetic might give wrong results. When on step 3 the algorithm checks whether two facet normals intersect (computing function $V(A ; v(D), v(E))$ ), any loss of precision may result in the wrong conclusion (if $d>2$ ). Therefore this algorithm is not numerically stable. When we know as a preliminary that a non-degenerate reciprocal exists, we can modify the algorithm so that it will construct an approximation 
of the reciprocal. Instead of computing the point of intersection of two lines (function $V(v(A) ; v(B), v(C)))$, a numerically stable version of Algorithm 1 should compute the point which is equidistant from both lines and minimizes the sum of the distances between a point and the lines. It does not increase the complexity of the algorithm, because it can be done in time $O(1)$. This can be also used for approximation of cell-decompositions of $\mathbb{R}^{d}$ which have a high proportion of primitive stars of $(d-2)$-cells by weighted Voronoi diagrams. Suzuki and Iri [41] report on how approximation of planar tesselations by Voronoi diagrams can be applied to urban planning and biological growth models.

What kind of manageable sufficient conditions can be used for determining whether a cell-decomposition of $\mathbb{R}^{d}(d>2)$ can be represented by a weighted diagram? Some such conditions are:

1. The decomposition is $(d-3)$-primitive (see Theorem 9.3).

2. The decomposition is $(d-2)$-primitive and for the star of every $(d-3)$-dimensional face there is an all-non-zero stress (see Theorem 9.2).

The second condition is not locally robust, but can be used for analysing tilings which consist of polytopes with symmetries arising from lattices (lattice polytopes, spacefillers) (see [36]).

\section{Algorithm for General Cell-Decompositions}

\subsection{Algorithm 2}

Let $M^{d}$ be stored as described in Section 10. The algorithm determines whether a lifting of a given type exists. Our strategy is to formulate a system of homogeneous linear equations and inequalities whose feasibility set coincides with the set of liftings of the prescribed type. For the variables of this system we take the $C_{1}^{0}$-cofactors of the stars of facets (see Sections 5, 10 and 11). The variables corresponding to those facets which are not involved in cycles of the dual graph are indeed free. Let $\left\{A\left(C_{j}\right)\right\}$ be a family of affine functions corresponding to the $d$-cells of $M^{d}$. By the results of Sections 4 and 5 if for each cycle $\mathfrak{c}_{i}=\left[C_{1}, \ldots, C_{k-1}, C_{1}\right]$ from a generating system $\left\{\mathfrak{c}_{i}\right\}$ of the cycle space of the dual graph $\mathcal{G}=(\mathcal{E}, \mathcal{V})$ of $\mathcal{M}^{d}$ the affine functions corresponding to $d$-cells of this cycle determine a lifting of the sub-complex which consists of $C_{1}, \ldots, C_{k-1}$, then $\left\{A\left(C_{j}\right)\right\}$ is a lifting of $M^{d}$. In terms of reciprocals it means that we have to pick edge lengths for a geometrical rectilinear realization of $\mathcal{G}$ so that edges are perpendicular to corresponding facets. It takes $O\left(f_{d-1}\right)$ operations to construct the dual graph $\mathcal{G}=(\mathcal{E}, \mathcal{V})$ from the graph of incidences of $\mathcal{M}^{d}$. The cost of finding a basis of the space of cycles for a graph with $|\mathcal{E}|$ edges is $O(|\mathcal{E}|)=O\left(f_{d-1}^{\circ}\right)$ operations (we find a spanning tree and then the basis associated with the tree). The number of cycles will be less than $|\mathcal{E}|$. In order to write equations expressing continuous gluing of $d$-cells of lifting over each cycle of the basis, we need to have these cycles oriented. It takes $O\left(|\mathcal{E}|^{2}\right)=O\left(f_{d-1}^{\circ 2}\right)$ operations. Note that by Lemma 4.2 if $H_{1}\left(\mathcal{M}^{d}, \mathbb{Z}_{2}\right)=0$, one can take the links of internal $(d-2)$-faces for a generating system of cycles. It requires $f_{d-2}^{\circ} f_{d-1}^{\circ}$ operations. This is reasonable when $d=2$, since the number of vertices in a planar tiling is less than the number of edges. If the facets of $M^{d}$ are situated in a general position relative 
to the coordinate system, the vanishing of the vector sum of cyclically ordered facet normals (scaled by the cofactors) in a $(d-2)$-cell $C^{d-2}$ can be expressed with two linear homogeneous scalar equations which involve only the two first coordinates of normals to facets sharing $C^{d-2}$ at $C^{d-2}$. This means that instead of taking $d f_{d-2}^{\circ}$ scalar equations, we can take only $2 f_{d-2}^{\circ}$. In this case the time complexity of the algorithm does not depend on $d$ at all.

Denote by $\Theta$ the system of $d\left(\operatorname{dim} H_{1}\left(\mathcal{G}, \mathbb{Z}_{2}\right)\right)$ scalar homogeneous equations corresponding to the basis elements of the space of cycles $H_{1}\left(\mathcal{G}, \mathbb{Z}_{2}\right)$ augmented with inequalities expressing restrictions on the qualitative type of lifting (like $x_{i}>0$ or $x_{i}<0$ or $\left.x_{i}=0\right)$. The complexity of constructing this system is $O\left(f_{d-1}^{2}\right)$. Thus the problem of finding a lifting of a given type is reduced to the problem of finding a solution for a system of $d f_{d-1}^{\circ}$ linear homogeneous equations and at most $f_{d-1}^{\circ}$ linear homogeneous strict inequalities with $f_{d-1}^{\circ}$ variables. These variables are $C_{1}^{0}$-cofactors of the stars of facets.

System $\Theta$ is a homogeneous feasibility problem in the standard Karmarkar form. Solutions to our original geometrical problem are represented by interior points of the cone $\Theta$. An interior point for the cone $\Theta$ can be found by recasting this problem to an auxiliary linear programming problem in the standard format and solving this auxiliary problem by a polynomial projective method (see [43]). A solution for $\Theta$ can also be effectively found via a modified Karmarkar algorithm for the homogeneous feasibility problem. The fact that the inequalities of $\Theta$ are strict does not affect the formal time complexity.

The time complexity of the algorithm will be given as a function of the number of $(d-1)$ - and $(d-2)$-cells. This estimation will also take into account the binary size of numerical input. Let $L$ be the binary size of the array of coordinates of normals to the supporting hyperplanes of facets. We assume that all operations are performed to a precision of $O(L \ln L)$. By Vaidya's estimates [43] the complexity of finding a feasible solution for the resulting feasibility problem by a modified projective (Karmarkar-like) method is $O\left(f_{d-1}^{\circ} L\right)$.

This leads to:

Theorem 12.1. Let $M^{d}$ be a PL-realization of a finite cell-decomposition of a manifold in $\mathbb{R}^{d}$. Algorithm 2 establishes whether $M^{d}$ has a lifting of a given type and finds such lifting in the case of the existence in time

$$
O\left(f_{d-1}^{\circ 3} L+f_{d-1}\right)
$$

If $H_{1}\left(\mathcal{M}^{d}, \mathbb{Z}^{2}\right)=0$, and the links of $(d-2)$-cells are used as a generating system, then the number of equations $f_{d-2}^{\circ}$ can be asymptotically larger than the number of variables $f_{d-1}^{\circ}$. Removing redundant equations by Gaussian elimination costs $O\left(f_{d-2}^{\circ} f_{d-1}^{\circ 2}\right)$ operations. After such reduction the number of equations does not exceed $f_{d-1}^{\circ}$. In this version the construction of $\Theta$ requires $O\left(f_{d-2}^{\circ} f_{d-1}^{\circ 2}+f_{d-2}^{\circ} f_{d-1}^{\circ}+f_{d-1}\right)$ operations. Thus the total time complexity of this version is

$$
O\left(f_{d-1}^{\circ 3} L+f_{d-2}^{\circ} f_{d-1}^{\circ 2}+f_{d-1}\right)
$$


where $L$ is the binary size of the array of the first two coordinates of normals to the supporting hyperplanes of facets.

Let $M^{d}$ be a realization of a homology sphere or a cell-decomposition of $\mathbb{R}^{d}$. It is interesting to estimate the complexity of the above algorithm in the case when the asymptotic upper bound theorem applies. Assume a sharp lifting for $M^{d}$ exists. (It is equivalent to the existence of an all-non-zero $d$-stress). In this case one can apply to $\mathcal{M}^{d}$ the asymptotic upper bound theorem, because even if the cell-decomposition of $\mathcal{M}^{d}$ is not simplicial or 0-primitive ( simple), one can prove the asymptotic upper bound via the perturbation of a sharp lifting. (For example, the asymptotic upper bound can always be used in the case $d=2$, since any cell-decomposition of a 2-manifold can be made simple by a small perturbation.) Let $n=f_{d}$ be the number of $d$-cells of $\mathcal{M}^{d}$. We have $f_{d-2}=O\left(n^{\min (\lfloor(d+1) / 2\rfloor, 3)}\right), f_{d-1}=O\left(n^{\min (\lfloor(d+1) / 2\rfloor, 2)}\right)$. Thus we have the following estimations for spherical manifolds: $O\left(n^{3} L\right)$ for $d=2$, and $O\left(n^{6} L\right)$ for $d \geq 3$.

\section{Fans}

It is natural to interpret all obtained results in terms of fans. A fan in Euclidean space $\mathbb{R}^{d}$ is a finite collection of pointed polyhedral cones which is closed under taking faces and intersections, and which covers $\mathbb{R}^{d}$. A fan can be alternatively regarded as a spherical complex, namely, a cell-decomposition of $\mathbb{S}^{d-1}$ where all cells are intersections of $\mathbb{S}^{d-1}$ and pointed polyhedral cones with centers at the center of $\mathbb{S}^{d-1}$. A convex polytope gives rise to its normal fan, whose cones are formed by normals to faces of the polytope. By analogy with the "flat" case, one can consider for a spherical complex $K$ radial liftings, i.e. polytopes such that their radial projections from the center of $\mathbb{S}^{d-1}$ give $K$. A star polytope generates a special fan. The apex of this fan is visible from any point of the boundary, and the cones of this fan are based on the faces of the polytope. We call such fans polytopical. Notice that a fan is the radial projection of a convex polytope if and only if this is a normal fan of a convex polytope. A fan is referred to as $k$-primitive if the star of each $k$-face has only $(d-k+1) d$-cones (minimal possible number). Alternatively, a spherical complex on $\mathbb{S}^{d-1}$ is referred to as $k$-primitive if the star of each $k$-face has exactly $d-k(d-1)$-cells.

Two questions are of interest:

1. Under what conditions is a given finite spherical complex $S^{d-1}$ the radial projection of the boundary complex of a convex polytope, or, in other words, when is a fan normal?

2. Under what conditions is a given finite spherical complex $S^{d-1}$ the radial projection of the boundary complex of a star polytope, or, in other words, when is a fan polytopical?

Denote by $\operatorname{StPol}(F)$ the manifold of star polytopes (up to the choice of a hyperplane) that have $F$ as their radial fan, and by $\operatorname{ConPol}(F)$ the cone of convex polytopes (in the same sense) that have this $F$ as their radial fan. These objects can be embedded into $\mathbb{R}^{f_{d-1}(F)-1}$.

All propositions in this section can be proved either via a straightforward adaptation of the arguments given for "flat" realizations to spherical complexes, or by regarding 
fans as finite cell-decompositions of $\mathbb{R}^{d}$ by convex polyhedra. In the latter case we lift a fan to a cone in $\mathbb{R}^{d+1}$, intersect this cone with a hyperplane parallel to $\mathbb{R}^{d}$, and project the intersection back onto $\mathbb{R}^{d}$. Depending on whether the cone is convex or not, the resulting polytope in $\mathbb{R}^{d}$ is either convex or star-like. By adopting the definition of a reciprocal (see Section 6) for the standard sphere $\mathbb{S}^{d-1}$ one can make use of this notion for spherical complexes.

Proposition 13.1. A fan is polytopical if and only if the spherical complex of the fan has a non-degenerate reciprocal. Convex polytopes correspond to convex reciprocals.

The theorem that a fan is normal if and only if the spherical complex of the fan has a convex reciprocal was proved earlier by McMullen [30]. We give here a characterization of fans of convex polytopes and star polytopes in terms of stresses. The definition of the $k$-stress for $k=1, \ldots, d-1$ (Section 3 ) works well for fans and spherical complexes. A $k$-stress on a fan corresponds to a $(k-1)$-stress on the corresponding spherical complex. Recall that, for manifolds with $H_{1}\left(\mathcal{M}^{d}, \mathbb{Z}_{2}\right)=0$, the coefficients of facet stresses can be regarded as $C_{1}^{0}$-cofactors of facets. In other words, they define affine forms which are the differences between functions representing a lifting on adjacent $d$-cells. The coefficients of a $d$-stress on facets of a fan can be interpreted in exactly the same way (see Theorem 5.1). Adopting the arguments from the proof of Theorem 5.1 for the spherical case, we have the following proposition.

Proposition 13.2. Let $F$ be a polyhedral fan in $\mathbb{R}^{d}(d>1)$. Then $\operatorname{dim} \operatorname{StPol}(F)=$ $\operatorname{dim}_{\text {Stress }}(F)$. Cones ConPol $(F)$ and Tension $_{d}(F)$ are isomorphic.

In other words, a fan in $\mathbb{R}^{d}$ is the radial fan of a convex (star) polytope if and only if there is a $d$-tension (all-non-zero $d$-stress) on this fan. The following propositions are adaptations of the results of Sections 3-11 for fans.

Proposition 13.3. Every $(d-3)$-primitive fan $(d>2)$ is the radial (normal) fan of a convex polytope which is unique up to the choice of a hyperplane and a dihedral angle.

Proposition 13.4. Let fan $F$ be $(d-2)$-primitive $(d>2)$. If there is an all-non-zero tension for each $(d-3)$-dimensional face of $F$, then $F$ is the radial (normal) fan of a convex polytope.

Proposition 13.5. Let $F$ be a $(d-2)$-primitive fan. There is an algorithm which determines whether $F$ is the radial (normal) fan of a convex polytope. This algorithm has linear time complexity in the number of $(d-1)$-cells of $F$. If such a convex polytope exists, it is unique up to the choice of a facet and a dihedral angle.

Proposition 13.6. There is a polynomial algorithm which determines whether a fan $F$ in $\mathbb{R}^{d}$ is the radial (normal) fan of a convex polytope. Let $n$ be the number of $d$-cones in $F$, and let $L$ be the binary size of an array of normals to $(d-1)$-cones of $F$. The 
time complexity of this algorithm is $O\left(n^{3} L\right)$ for $d=3$, and $O\left(n^{6} L\right)$ if $d>3$ and the asymptotic upper bound theorem applies.

In [37] Shephard gave necessary and sufficient conditions in terms of Gale diagrams that a given finite spherical complex $S^{d-1}$ is the radial projection of the boundary complex of a convex polytope. Aurenhammer [5], [7] worked out applications of this approach to scene analysis and Voronoi diagrams. We can interpret the Gale diagram technique in terms of computational complexity. Our analysis will be based on the assumption that the algorithm works with a data structure which is well adapted for it. For example, we can assume that the nodes representing the rays of a fan $F$ in the incidence graph of $F$ contain their coordinates. Notice that Algorithm 2 requires inequalities determining $(d-1)$-cells of $F$, whereas an algorithm which can be extracted from the Shephard theorem requires coordinates of rays of $F$. If $f_{k}$ is the number of $k$-cells of $S^{d-1}((k+1)$-cones of $F)$, then Shephard's condition is the existence of an interior point in the intersection of a finite number of convex polytopes in $\mathbb{R}^{f_{0}-d}$ (see [37] and [38]). Each polytope of this family corresponds to a $(d-1)$-cell of $S^{d-1}$. If a $(d-1)$-cell $C_{i}^{d-1}$ has $f_{0}\left(C_{i}^{d-1}\right)$ vertices, then the corresponding polytope $G\left(C_{i}^{d-1}\right)$ is the convex hall of $f_{0}-f_{0}\left(C_{i}^{d-1}\right)$ vertices. Switching to dual polytopes $G^{*}\left(C_{i}^{d-1}\right)$ one can see that the problem has polynomial complexity. Let $\bigcap_{i=1}^{f_{d-1}} G^{*}\left(C_{i}^{d-1}\right)$ be the intersection of polytopes $G^{*}\left(C_{i}^{d-1}\right)$ over all $(d-1)$-cells. Notice that unlike system $\Theta$ from Algorithm 2 affine equations determining the polytope $\bigcap_{i=1}^{f_{d-1}} G^{*}\left(C_{i}^{d-1}\right)$ in Shephard's criterion are not homogeneous. The complexity of solving the feasibility problem for $\bigcap_{i=1}^{f_{d-1}} G^{*}\left(C_{i}^{d-1}\right)$ can be formally estimated as $O\left(\left(\left(f_{0} f_{d-1}+\right.\right.\right.$ $\left.\left.\left.f_{0}\right) f_{0}^{2}+\left(f_{0} f_{d-1}+f_{0}\right)^{1.5} f_{0}\right) L\right)$ in the worst case ( $L$ is the binary size of numerical input) [43]. For example, for $d=3$ it gives $O\left(f_{0}^{4} L\right)=O\left(f_{d-1}^{4} L\right)$ which is asymptotically worse than $O\left(f_{d-1}^{3} L\right)$ given by Proposition 13.6.

\section{Acknowledgments}

The author is grateful for many stimulating conversations with R. M. Erdahl, S. S. Ryshkov and W. Whiteley.

\section{References}

1. P. Ash and E. Bolker, Generalized Dirichlet tessellations, Geom. Dedicata 20 (1986), 209-243.

2. P. Ash, E. Bolker, H. Crapo and W. Whitely, Convex polyhedra, Dirichlet tessellations, and spider webs, in Shaping Space: A Polyhedral Approach, M. Senechal and G. Fleck, eds., Boston: Birkhäuser, 1988, pp. 231-250.

3. F. Aurenhammer, Recognizing polytopical cell complexes and constructing projection polyhedra, J. Symbolic Comput. 3 (1987), 249-255.

4. F. Aurenhammer, A criterion for the affine equivalence of cell complexes in $\mathbb{R}^{d}$ and convex polyhedra in $R^{d+1}$, Discrete Comput. Geom. 2 (1987), 49-64.

5. F. Aurenhammer, A relationship between Gale transforms and Voronoi diagrams, Discrete Appl. Math. 28 (1990), 83-91.

6. F. Aurenhammer, Voronoi diagrams: a survey of a fundamental data structure, ACM Comput. Surveys $\mathbf{2 3}$ (1991), 345-405. 
7. F. Aurenhammer, Using Gale transforms in computational geometry, Math. Programming 52 (1991), 179-190.

8. L. Billera, Homology of smooth splines: generic triangulations and a conjecture of Strang, Trans. Amer. Math. Soc. 310 (1988), 325-340.

9. L. Billera, Algebra of continuous piecewise polynomials, Adv. in Math. 76 (1989), 170-183.

10. R. Connelly and D. W. Henderson, A convex 3-complex not simplicially isomorphic to a strictly convex complex, Math. Proc. Cambridge Philos. Soc. 88(2) (1980), 299-306.

11. R. Connelly and W. Whiteley, Second-order rigidity and prestress stability for tensegrity frameworks, SIAM J. Discrete Math. 9(3) (1996), 453-491.

12. H. Crapo and W. Whiteley, Plane stresses and projected polyhedra, 1: the basic pattern. Structural Topology 19 (1993), 55-78.

13. H. Crapo and W. Whiteley, Spaces of stresses, projections and parallel drawings for spherical polyhedra, Contrib. Algebra Geom. 35(2) (1994), 259-281.

14. H. Crapo and W. Whiteley, 3-Stresses in 3-space and projections of 4-polytopes: reciprocals, liftings and parallel configurations; Preprint, York University, North York, Ontario, 1994.

15. C. Davis, The set of non-linearity of a convex piecewise-linear function, Scripta Math. 24 (1959), 219-228.

16. B. N. Delaunay [Delone], Sur la sphère vide, Proceedings of the International Congress of Mathematicians, Toronto, 1924, University of Toronto Press, Toronto, 1928, pp. 695-700.

17. B. N. Delaunay [Delone], Sur ls partition reǵulier̀e de l'espace a 4 dimensions, Bull. Acad. Sci. URSS (1929), No. 1, 79-110, No. 2, 147-171.

18. B. N. Delone, The geometry of positive quadratic forms, Uspekhi Mat. Nauk 3 (1937), 16-62; 4 (1938), 102-164.

19. B. N. Delone, Peterburgskaja shkola teorii chisel [The Petersbourgh's School of Number Theory] (in Russian) Leningrad: Nauka, 1947.

20. H. Edelsbrunner, Algorithms in Combinatorial Geometry, New York: Springer-Verlag, 1987.

21. H. Edelsbrunner, The union of balls and its dual shape, Discrete Comput. Geom. 13(3-4) (1996), 415-440.

22. H. Edelsbrunner and N. Shah, Incremental topological flipping works for regular triangulations, Algorithmica 15 (1996), 223-241.

23. R. M. Erdahl, Dicings and Voronoi conjecture on parallelohedra, European J. Combin., to appear.

24. R. M. Erdahl, K. A. Rybnikov and S. S. Ryshkov, Traces of $d$-stresses in skeletons of lower dimensions of homology $d$-manifolds, Preprint, Queen's University, Kingston, Ontario, 1997.

25. H. Imai, On combinatorial structures of line drawings of polyhedra, Discrete Appl. Math. 9 (1984), 47-67.

26. C. W. Lee, P.L.-spheres, convex polytopes, and stress, Discrete Comput. Geom. 15 (1996), 389-421.

27. S. Lefschetz, Algebraic Topology, Providence, RI: American Mathematical Society, 1942.

28. J. C. Maxwell, On reciprocal diagrams and diagrams of forces, Phil. Mag. Ser. 427 (1864), 250-261.

29. J. C. Maxwell, On reciprocal diagrams, frames and diagrams of forces, Trans. Roy. Soc. Edinburgh 26 (1869-72), 1-40.

30. P. McMullen, Representations of polytopes and polyhedral sets, Geom. Dedicata 2 (1973), 83-99.

31. P. McMullen, On simple polytopes, Invent. Math. 113(2) (1993), 419-444.

32. P. McMullen, Duality, sections and projections of certain Euclidean tilings, Geom. Dedicata 49(2) (1994), 183-202.

33. P. McMullen, Weights on polytopes, Discrete Comput. Geom. 15 (1996), 363-388.

34. J. R. Munkres, Elements of Algebraic Topology, Reading, MA: Addison-Wesley, 1984.

35. S. S. Ryshkov and K. A. Rybnikov, Jr., Generatrissa: the problems of Maxwell and Voronoi, Dokl. Math. 54(1) (1996), 614-617.

36. S. S. Ryshkov and K. A. Rybnikov, Jr., The theory of quality translation with applications to tilings, European J. Combin. 18(4) (1997), 431-445.

37. G. C. Shephard, Diagrams for positive bases, J. London Math. Soc. (2) 4 (1971), 165-175.

38. G. C. Shephard, Spherical complexes and radial projections of polytopes, Israel J. Math. 9 (1971), 257262.

39. K. Sugihara, Machine Interpretation of Line Drawings, Cambridge, MA: MIT Press, 1986.

40. K. Sugihara, A. Okabe and B. Boots, Spatial Tesselations: Concepts and Applications of Voronoi Diagrams, Chichester: Wiley, 1992.

41. A. Suzuki and M. Iri, Approximation of a tesselation of the plane by a Voronoi diagram, J. Oper. Res. Soc. Japan 29(1) (1986), 69-96. 
42. T.-S. Tay, N. White and W. Whiteley, Skeletal rigidity of simplicial complexes, 1, 2, European J. Combin. 16 (1995), 381-403, 503-523.

43. P. M. Vaidya, An algorithm for linear programming which requires $O\left(\left((m+n) n^{2}+(m+n)^{1.5} n\right) L\right)$ arithmetic operation, Math. Programming 47 (1990), 175-202.

44. G. F. Voronoi, Nouvelles applications des paramèters continus à la théorie des formes quadratiques, Deuxième mémoire, J. Reine Angew. Math 134 (1908), 198-287, 136 (1909), 67-178.

45. W. Whiteley, Motions and stress of projected polyhedra, Structural Topology 7 (1982), 13-38.

46. W. Whiteley, Two algorithms for polyhedral pictures, Proceedings of the 2 nd Annual ACM Symposium on Computational Geometry, New York, 1986, pp. 142-149.

47. W. Whiteley, A matroid on hypergraphs with applications to scene analysis and geometry, Discrete Comput. Geom. 4 (1989), 75-95.

48. W. Whiteley, 3-Diagrams and Schlegel diagrams of simple 4-polytopes, Preprint, 1994.

49. W. Whiteley, How to describe or design a polyhedron, J. Intel. Robotic Systems 11 (1994), 135-160.

50. W. Whiteley, Matroids from Discrete Geometry, Contemporary Mathematics, Vol. 197, 1996, pp. 171-312.

51. O. Zhitomirski, Verscharfung eines Satzes vom Woronoi, Z. Leningrad. Fiz.-Mat. Obsc. 2 (1929), 131-151.

52. G. Ziegler, Lectures on Polytopes, New York: Springer-Verlag, 1995.

Received April 24, 1997, and in revised form July 31, 1998. 